\title{
Performance and Optimization of Network-Coded Cooperative Diversity Systems ${ }^{1}$
}

\author{
Amir Nasri ${ }^{\dagger}$, Robert Schober ${ }^{\dagger}$, and Murat Uysal ${ }^{\dagger \dagger}$ \\ Department of Electrical and Computer Engineering \\ †University of British Columbia, Canada, †'University of Waterloo, Canada
}

In this paper, we study network-coded cooperative diversity (NCCD) systems comprising multiple sources, one relay, and one destination, where the relay detects the packets received from all sources and performs Galois field network coding before forwarding a single packet to the destination. We develop a simple generalized cooperative maximum-ratio combining scheme for the destination which achieves a similar performance as optimal maximum-likelihood combining. Furthermore, assuming independent Rayleigh fading for all links of the network, we provide a mathematical framework for the analysis of the error performance of NCCD systems in the high signal-to-noise ratio regime. Based on this framework, we derive simple and elegant closed-form expressions for the asymptotic symbol and bit error rates of NCCD systems. The derived error rate expressions are valid for arbitrary numbers of sources, arbitrary modulation schemes, and arbitrary constellation mappings and provide significant insight into the impact of various system and channel parameters on performance. These expressions can also be exploited for optimization of the constellation mapping as well as for formulation of various NCCD system optimization problems including optimal power allocation, relay selection, and relay placement. Simulation results confirm the accuracy of the presented analysis and reveal that the performance of NCCD systems can be considerably improved by optimizing the constellation mapping and the power allocation based on the developed analytical results.

\footnotetext{
${ }^{1}$ This work will be presented in part at the IEEE Global Telecommunications Conference (Globecom), Miami, 2010 .
} 


\section{Introduction}

Cooperative diversity $(C D)$ is an effective technique to exploit the spatial diversity offered by wireless relay nodes. However, since the cooperating terminals typically use orthogonal channels for transmission to simplify processing at the relays and the destination, CD entails a throughput reduction $[1,2]$. This throughput reduction is most noticeable in CD systems with multiple source terminals since in such systems the relays use separate orthogonal channels to forward the signals received from different sources. As a result, the relays can serve only a single source in a given time or frequency slot, and therefore the available resources are not shared efficiently by different sources.

Network coding over Galois fields (GFs) is an efficient approach to increase the throughput of multi-source CD systems [3]-[5]. The idea of network coding was originally developed for wired networks as an efficient routing technique capable of enhancing the network throughput [6]. In the context of $\mathrm{CD}$, network coding can be employed to overcome the associated throughput bottleneck by allowing relays to simultaneously serve multiple source terminals.

The combination of CD and GF network coding, which we refer to as network-coded CD (NCCD) in this work, has received considerable attention recently. In particular, the outage capacity of NCCD systems was calculated in [3, 4], and their diversity-multiplexing tradeoff was analyzed in [5]. In [7], for a network coding system employing an algebraic superposition of channel codes and iterative decoding at the destination, optimal channel codes were designed based on an ad-hoc code search. The diversity order of an NCCD system employing distributed error-correcting codes was analyzed in [8], and it was shown that a maximum diversity order equal to the minimum distance of the employed error-correcting code can be achieved. Also, physical-layer network coding (PNC) [9] and complex field network coding (CFNC) [10] have been proposed as interesting alternatives to NCCD. However, unlike NCCD, for both of these schemes the relay receives the transmissions of multiple sources simultaneously, which makes time and frequency synchronization very challenging. Furthermore, the relay transmit signals for PNC and CFNC do not belong to a standard signal constellation and, as a result, may suffer from a high peak-to-average power ratio.

While error rate expressions which facilitate performance evaluation and system optimization are desirable, existing works on NCCD systems [3]-[8] do not provide a general and accurate error rate analysis. Furthermore, these works assume network coding in the GF of order two, and therefore do not explore potential benefits of notwork coding over GFs of higher order. Thus, in this paper, we investigate the error rate performance of NCCD systems comprising multiple sources, one relay, and one destination, where network coding is performed over the GF of order $M=2^{m}$ and an arbitrary $M$-ary modulation is empolyed by the sources and the relay. Furthermore, in order to obtain a simple combining scheme at the destination, which is amenable to analysis and achieves a similar performance as optimal maximum-likelihood (ML) combining, we generalize cooperative maximum- 
ratio combining (C-MRC), which was proposed in [11] for conventional CD systems, to NCCD. For the resulting NCCD system we derive simple and elegant closed-form expressions for the asymptotic symbol and bit error rates in Rayleigh fading. These closed-form expressions give valuable insight into the impact of various system and channel parameters (e.g., the number of sources, the signal-to-noise ratios (SNRs) of the involved wireless links, the signal constellation, and the constellation mapping) on performance. For example, our analytical results reveal that the achieved diversity gain for all source terminals is equal to two irrespective of the number of sources. In contrast, the network-coding gain is source dependent and is affected by various system and channel parameters. Furthermore, the developed error rate expressions can be exploited for various NCCD system optimization problems including optimal constellation mapping, power allocation, relay selection, and relay placement.

The remainder of this paper is organized as follows. In Section 2, the some notations and definitions and the system model of the considered NCCD system are introduced. Accurate asymptotic expressions for the symbol error rate (SER) and the bit error rate (BER) of NCCD systems are derived in Section 3. Optimal power allocation for NCCD systems is discussed in Section 4, and numerical and simulation results are presented in Section 5. Finally, some conclusions are drawn in Section 6.

\section{Preliminaries}

In this section, we describe the model for the considered NCCD system and introduce some notations and definitions.

\subsection{Notations and Definitions}

In this paper, $[\cdot]^{T},(\cdot)^{*}, \Re\{\cdot\}, \mathcal{E}_{x}\{\cdot\}, \Gamma(\cdot), \Gamma(\cdot, \cdot)$, and $\psi(\cdot)$ denote transposition, complex conjugation, the real part of a complex number, statistical expectation with respect to $x$, the Gamma function, the upper incomplete Gamma function, and the Digamma function, respectively. $Q(x) \triangleq \frac{1}{\sqrt{2 \pi}} \int_{x}^{\infty} \mathrm{e}^{-t^{2} / 2} \mathrm{~d} t$ denotes the Gaussian $Q$-function. Furthermore, we use the notation $u \stackrel{\ominus}{=}$ to indicate that $u$ and $v$ are asymptotically equivalent, and a function $f(x)$ is $o(g(x))$ if $\lim _{x \rightarrow 0} f(x) / g(x)=0$.

\subsection{Signal Model}

The considered NCCD system is depicted in Fig. 1 and comprises $N_{s}$ source terminals $S_{i}, 1 \leq i \leq N_{s}$, one relay $R$, and one destination terminal $D$. Transmission from the source terminals to the destination terminal is organized in two hops. The first hop comprises $N_{s}$ orthogonal time or frequency slots (referred to as channel slots in the following), where each source terminal $S_{i}, 1 \leq i \leq N_{s}$, transmits its message to the relay and the destination. In particular, a data symbol $s_{i} \in \mathcal{A}$ is generated at source $S_{i}$, where $\mathcal{A} \triangleq \mathrm{GF}\left(2^{m}\right)$ is the GF of order $M=2^{m}$. This data symbol is mapped to a 
transmit symbol $x_{i} \in \mathcal{X}$ with $\mathcal{E}\left\{\left|x_{i}\right|^{2}\right\}=1$ using the mapping $x_{i}=\mu_{\mathcal{X}}\left(s_{i}\right)$, where $\mathcal{X}$ denotes an $M-$ ary signal constellation such as $M$-ary phase-shift keying ( $M$-PSK) or $M$-ary quadrature amplitude modulation ( $M-\mathrm{QAM})$, and $\mu_{\mathcal{X}}: \mathcal{A} \rightarrow \mathcal{X}$ is a one-to-one constellation mapping function from $\mathcal{A}$ to $\mathcal{X}$. Subsequently, source $S_{i}$ transmits symbol $x_{i}$ to the relay and the destination. The signals received by the destination and the relay in the first hop, $r_{S_{i} D}$, are given by

$$
r_{S_{i} D}=\sqrt{P_{i}} f_{i} x_{i}+n_{D, i} \quad \text { and } \quad r_{S_{i} R}=\sqrt{P_{i}} g_{i} x_{i}+n_{R, i}, \quad 1 \leq i \leq N_{s}
$$

respectively, where $P_{i}$ is the average transmit power of the $i$ th source, and $f_{i}$ and $g_{i}$ denote the fading gains of the $S_{i} \rightarrow D$ and the $S_{i} \rightarrow R$ channels, respectively. Furthermore, $n_{D, i}$ and $n_{R, i}$ denote the additive white Gaussian noise (AWGN) samples at the destination and the relay with variances $\sigma_{n_{D, i}}^{2} \triangleq \mathcal{E}\left\{\left|n_{D, i}\right|^{2}\right\}$ and $\sigma_{n_{R, i}}^{2} \triangleq \mathcal{E}\left\{\left|n_{R, i}\right|^{2}\right\}$, respectively.

The relay performs coherent $\mathrm{ML}$ detection and generates the detected symbols

$$
\hat{x}_{R, i}=\arg \min _{\tilde{x} \in \mathcal{X}}\left\{\left|r_{S_{i} R}-\sqrt{P_{i}} g_{i} \tilde{x}\right|^{2}\right\}, \quad 1 \leq i \leq N_{s},
$$

which correspond to detected data symbols $\hat{s}_{R, i}=\mu_{\mathcal{X}}^{-1}\left(\hat{x}_{R, i}\right) \in \mathcal{A}, 1 \leq i \leq N_{s}$.

The second hop comprises a single channel slot. In particular, in the second hop the relay performs network coding and computes the data symbol $\hat{s}_{R} \triangleq \hat{s}_{R, 1} \oplus \cdots \oplus \hat{s}_{R, N_{s}} \in \mathcal{A}$, where $\oplus$ denotes addition in $\operatorname{GF}\left(2^{m}\right)$. The relay then forwards the transmit symbol $\hat{x}_{R} \triangleq \mu_{\mathcal{X}}\left(\hat{s}_{R}\right) \in \mathcal{X}$ to the destination. The signal received at the destination in the second hop, $r_{R D}$, can be modeled as

$$
r_{R D}=\sqrt{P_{R}} h_{R} \hat{x}_{R}+n_{D, R},
$$

where $P_{R}$ is the average transmit power of the relay, $h_{R}$ is the fading gain of the $R \rightarrow D$ channel, and $n_{D, R}$ is the AWGN at the destination in the second hop having variance $\sigma_{n_{D, R}}^{2} \triangleq \mathcal{E}\left\{\left|n_{D, R}\right|^{2}\right\}$.

Throughout this paper we assume independent Rayleigh fading for all links of the network. Thus, the fading gains $f_{i} \triangleq a_{f_{i}} e^{-j \theta_{f_{i}}}, g_{i} \triangleq a_{g_{i}} e^{-j \theta_{g_{i}}}, 1 \leq i \leq N_{s}$, and $h_{R} \triangleq a_{h_{R}} e^{-j \theta_{h_{R}}}$ are independent Gaussian random variables (RVs) with zero mean and variances $\Omega_{f_{i}} \triangleq \mathcal{E}\left\{\left|f_{i}\right|^{2}\right\}, \Omega_{g_{i}} \triangleq \mathcal{E}\left\{\left|g_{i}\right|^{2}\right\}$, $1 \leq i \leq N_{s}$, and $\Omega_{R} \triangleq \mathcal{E}\left\{\left|h_{R}\right|^{2}\right\}$, respectively. Here, the channel amplitudes $a_{f_{i}}, a_{g_{i}}$, and $a_{h_{R}}$ are positive real RVs and follow a Rayleigh distribution. Furthermore, the channel phases $\theta_{f_{i}}, \theta_{g_{i}}$, and $\theta_{h_{R}}$ are uniformly distributed in $[-\pi, \pi)$ and are independent from the channel amplitudes.

For future reference, we define the instantaneous SNRs of the $S_{i} \rightarrow D, S_{i} \rightarrow R$, and $R \rightarrow D$ links as $\gamma_{f_{i}} \triangleq P_{i} a_{f_{i}}^{2} / \sigma_{n_{D, i}}^{2}, \gamma_{g_{i}} \triangleq P_{i} a_{g_{i}}^{2} / \sigma_{n_{R, i}}^{2}$, and $\gamma_{h_{R}} \triangleq P_{R} a_{h_{R}}^{2} / \sigma_{n_{R}}^{2}$, respectively. The corresponding average SNRs are given by $\bar{\gamma}_{f_{i}}=P_{i} \Omega_{f_{i}} / \sigma_{n_{D, i}}^{2}, \bar{\gamma}_{g_{i}}=P_{i} \Omega_{g_{i}} / \sigma_{n_{R, i}}^{2}$, and $\bar{\gamma}_{D, R}=P_{R} \Omega_{R} / \sigma_{n_{D, R}}^{2}$, respectively.

Remark 1: Based on the presented signal model, a total of $N_{s}+1$ channel slots are required for transmission of the signals of all $N_{s}$ sources to the destination. In contrast, a conventional CD system $[1,11]$ requires $2 N_{s}$ channel slots since the relay assists only a single source in a given channel slot. 


\subsection{Equivalent Source-Relay Channel}

In this subsection, we introduce an equivalent channel between the source terminals and the relay for the considered NCCD system which will be particularly useful for developing the diversity combining scheme in Section 2.4 and the performance analysis in Section 3. The input of this equivalent channel, $x_{R}$, is the relay transmit symbol in the absence of noise, i.e., $x_{R} \triangleq \mu_{\mathcal{X}}\left(s_{R}\right) \in \mathcal{X}$ with $s_{R} \triangleq s_{1} \oplus \cdots \oplus s_{N_{s}} \in \mathcal{A}$, and the output is the actual relay transmit symbol $\hat{x}_{R}$. Defining the source-relay SNR vector $\gamma_{g} \triangleq\left[\gamma_{g_{1}}, \cdots, \gamma_{g_{N_{s}}}\right]^{T}$, this channel is characterized by the equivalent error probability $P_{e, \text { eq }}\left(\gamma_{g}\right) \triangleq \operatorname{Pr}\left\{\hat{x}_{R} \neq x_{R}\right\}$. For an $M$-ary signal constellation $\mathcal{X}$, the equivalent error probability $P_{e, \text { eq }}\left(\gamma_{g}\right)$ can be approximated by $P_{e, \text { eq }}\left(\gamma_{g}\right)=\beta Q\left(\sqrt{2 \alpha \gamma_{\text {eq }}\left(\gamma_{g}\right)}\right)$, where $\alpha$ and $\beta$ are two modulation dependent constants (e.g. $\alpha=\beta=1$ for BPSK). Furthermore, $\gamma_{\mathrm{eq}}\left(\gamma_{g}\right)$ is the instantaneous SNR associated with the equivalent source-relay channel which can be expressed as $\gamma_{\text {eq }}\left(\gamma_{g}\right)=\frac{1}{2 \alpha}\left(Q^{-1}\left(P_{e, \text { eq }}\left(\gamma_{g}\right) / \beta\right)\right)^{2}$. It can be shown that for sufficiently high SNR $\gamma_{\text {eq }}\left(\gamma_{g}\right)$ can be accurately approximated as $\gamma_{\mathrm{eq}}\left(\gamma_{g}\right)=\min \left\{\gamma_{g_{1}}, \cdots, \gamma_{g_{N_{s}}}\right\}$. As a result, since $\gamma_{g_{i}}, 1 \leq i \leq N_{s}$, is an exponentially distributed RV with mean $\bar{\gamma}_{g_{i}}, \gamma_{\text {eq }}\left(\gamma_{g}\right)$ is also exponentially distributed with mean $\bar{\gamma}_{\text {eq }}=\left(1 / \bar{\gamma}_{g_{1}}+\cdots+1 / \bar{\gamma}_{g_{N_{s}}}\right)^{-1}$. In the following, we use $\gamma_{\mathrm{eq}}$ instead of $\gamma_{\mathrm{eq}}\left(\boldsymbol{\gamma}_{g}\right)$ for simplicity of notation.

\subsection{Diversity Combining at the Destination}

$\mathrm{ML}$ combining can be employed at the destination to optimally combine the signals received from the sources and the relay. However, due to the possibility of erroneous decisions at the relay, the ML decision metric is complex and not amenable to analysis. In order to avoid the problems associated with the ML metric, we generalize the C-MRC scheme proposed in [11] for conventional CD to NCCD. As will be shown in Sections 3 and 5, the simple C-MRC scheme performs close to the ML combining and exploits the full diversity of NCCD systems for any number of sources. The proposed generalized $\mathrm{C}-\mathrm{MRC}$ metric is given by

$$
m_{c}(\tilde{\boldsymbol{x}})=\sum_{i=1}^{N_{s}} \frac{\left|r_{S_{i} D}-\sqrt{P_{i}} f_{i} \tilde{x}_{i}\right|^{2}}{\sigma_{n_{D, i}}^{2}}+\lambda_{R} \frac{\left|r_{R D}-\sqrt{P_{R}} h_{R} \tilde{x}_{R}\right|^{2}}{\sigma_{n_{D, R}}^{2}}
$$

Here, vector $\tilde{\boldsymbol{x}} \triangleq\left[\tilde{x}_{1} \ldots \tilde{x}_{N_{s}}\right]^{T} \in \mathcal{X}^{N_{s}}$ contains trial transmit symbols $\tilde{x}_{i}=\mu_{\mathcal{X}}\left(\tilde{s}_{i}\right) \in \mathcal{X}, 1 \leq i \leq N_{s}$, where $\tilde{s}_{i} \in \mathcal{A}, 1 \leq i \leq N_{s}$, are trial data symbols. Furthermore, in (4) we have introduced $\tilde{x}_{R} \triangleq \mu_{\mathcal{X}}\left(\tilde{s}_{R}\right) \in \mathcal{X}$ with $\tilde{s}_{R} \triangleq \tilde{s}_{1} \oplus \cdots \oplus \tilde{s}_{N_{s}} \in \mathcal{A}$ and the weighting factor $\lambda_{R} \triangleq \frac{\min \left\{\gamma_{\text {eq }}, \gamma_{R}\right\}}{\gamma_{R}} \in[0,1]$. For the case that all $S_{j} \rightarrow R$ channels have higher SNRs than the $R \rightarrow D$ channel, $\lambda_{R}=1$ is valid and (4) reduces to conventional MRC. However, if at least one of the $S_{j} \rightarrow R$ channels has a poorer quality than the $R \rightarrow D$ channel, the metric in (4) assigns a smaller weight $\lambda_{R}<1$ to the part of the metric associated with the signal received from the relay in order to take into account the effect 
of possibly erroneous decisions at the relay. In order to compute $\lambda_{R}$, the destination has to know the SNR of the weakest source-relay channel. This SNR can be estimated at the relay, which has to know the corresponding channel gain for coherent detection, and be forwarded to the destination over a low-rate feedback channel.

Based on (4) signal detection at the destination can be performed as $\hat{\boldsymbol{x}}_{D}=\arg \min _{\tilde{\boldsymbol{x}} \in \mathcal{X}^{N_{s}}}\left\{m_{c}(\tilde{\boldsymbol{x}})\right\}$, where $\hat{\boldsymbol{x}}_{D} \triangleq\left[\hat{x}_{D, 1} \ldots \hat{x}_{D, N_{s}}\right]^{T} \in \mathcal{X}^{N_{s}}$ contains the detected symbols at the destination for all sources and the corresponding decoded data symbols are obtained as $\hat{s}_{D, i} \triangleq \mu_{\mathcal{X}}^{-1}\left(\hat{x}_{D, i}\right) \in \mathcal{A}, 1 \leq i \leq$ $N_{s}$. Brute force determination of $\hat{\boldsymbol{x}}_{D}$ requires $M^{N_{s}}$ metric computations, i.e., complexity increases exponentially with $N_{s}$. However, detection complexity can be significantly reduced by exploiting the fact that the data vectors $\boldsymbol{s}_{e} \triangleq\left[s_{1}, \cdots, s_{N_{s}}, s_{R}\right]^{T} \in \mathcal{A}^{N_{s}+1}$ form an $\left(N_{s}+1, N_{s}\right)$ single-paritycheck block code over $\operatorname{GF}\left(2^{m}\right)$. As a result, the signal detection at the destination can be efficiently implemented using well-known soft-decision decoding algorithms for block codes from the literature [12], e.g. Viterbi decoding based on the trellis representation of the corresponding single-parity-check block code [13]. However, a detailed discussion of such algorithms is beyond the scope and the page limits of the current paper.

\section{Performance Analysis}

In this section, we analyze the error rate performance of the considered NCCD system for high SNRs, i.e., $\bar{\gamma}_{f_{i}}, \bar{\gamma}_{g_{i}} \rightarrow \infty, 1 \leq i \leq N_{s}$, and $\bar{\gamma}_{R} \rightarrow \infty$. In particular, we develop accurate asymptotic closedform expressions for the pairwise error probabilities (PEPs), SERs, and BERs of all sources. For convenience, we introduce the source-destination SNR vector $\gamma_{f} \triangleq\left[\gamma_{f_{1}}, \cdots, \gamma_{f_{N_{s}}}\right]^{T}$, the normalized noise samples $\bar{n}_{D, i} \triangleq n_{D, i} / \sigma_{n_{D, i}}, 1 \leq i \leq N_{s}$, and $\bar{n}_{D, R} \triangleq n_{D, R} / \sigma_{n_{D, R}}$, and the noise vector $\boldsymbol{n} \triangleq\left[\bar{n}_{D, 1}, \cdots, \bar{n}_{D, N_{s}}, \bar{n}_{D, R}\right]^{T}$.

Using a union bound over the pairwise error probabilities, for the $i$ th source, the SER, $P_{s}^{i}$, can be upper-bounded as

$$
P_{s}^{i} \leq \frac{1}{M^{N_{s}}} \sum_{\boldsymbol{x} \in \mathcal{X}^{N_{s}}} \sum_{\tilde{\boldsymbol{x}} \in \mathcal{B}_{i}(\boldsymbol{x})} P(\boldsymbol{x} \rightarrow \tilde{\boldsymbol{x}}),
$$

where $P(\boldsymbol{x} \rightarrow \tilde{\boldsymbol{x}})$ denotes the PEP associated with the pair $(\boldsymbol{x}, \tilde{\boldsymbol{x}})$ which is the probability that $\boldsymbol{x} \triangleq\left[x_{1} \cdots x_{N_{s}}\right]^{T} \in \mathcal{X}^{N_{s}}$ was transmitted by the sources and $\tilde{\boldsymbol{x}}=\left[\tilde{x}_{1} \cdots \tilde{x}_{N_{s}}\right]^{T} \in \mathcal{X}^{N_{s}}, \tilde{\boldsymbol{x}} \neq \boldsymbol{x}$, was detected at the destination assuming that $\boldsymbol{x}$ and $\tilde{\boldsymbol{x}}$ are the only possible decision outcomes. The set $\mathcal{B}_{i}(\boldsymbol{x})$ in (5) is defined as

$$
\mathcal{B}_{i}(\boldsymbol{x}) \triangleq\left\{\tilde{\boldsymbol{x}} \mid \tilde{x}_{j} \in \mathcal{X} \backslash\left\{x_{j}\right\}, j=i, \tilde{x}_{j} \in \mathcal{X}, j \neq i\right\} .
$$

In the following, we first derive an asymptotic expression for the PEP in Subsection 3.1 before we use an expurgated version of the union bound in (5) to arrive at an accurate closed-form expressions for the asymptotic SER and BER in Subsection 3.2. 


\subsection{Asymptotic Pairwise Error Probability}

The PEP for the considered NCCD system can be expressed as

$$
P(\boldsymbol{x} \rightarrow \tilde{\boldsymbol{x}})=\operatorname{Pr}\left\{m_{c}(\boldsymbol{x})>m_{c}(\tilde{\boldsymbol{x}})\right\}
$$

It is convenient to calculate first the PEP conditioned on the instantaneous SNRs $\left(\gamma_{f}, \gamma_{g}, \gamma_{R}\right)$ and noise vector $\boldsymbol{n}$. To obtain such an expression, we assume that among the transmit symbols $x_{j}$, $1 \leq j \leq N_{s}$, at most one is received in error at the relay. Furthermore, we assumed that if transmit symbol $x_{j}$ is received in error, the erroneous $\hat{x}_{R, j}$ at the relay is a nearest neighbor of $x_{j}$, i.e., $\hat{x}_{R, j} \in \mathcal{N}\left(x_{j}\right)$, where set $\mathcal{N}(x)$ contains all nearest neighbors of $x$ in $\mathcal{X}$. The approximations related to these assumptions are well justified for $\bar{\gamma}_{g_{j}} \rightarrow \infty, 1 \leq j \leq N_{s}$, and their accuracy will be confirmed by simulations in Section 5. The desired conditional PEP can now be expressed as

$$
\begin{aligned}
P\left(\boldsymbol{x} \rightarrow \tilde{\boldsymbol{x}} \mid \boldsymbol{\gamma}_{f}, \boldsymbol{\gamma}_{g}, \gamma_{R}, \boldsymbol{n}\right) & =\operatorname{Pr}\left\{\hat{x}_{R}=x_{R}\right\} P\left(\boldsymbol{x} \rightarrow \tilde{\boldsymbol{x}} \mid x_{R}, \boldsymbol{\gamma}_{f}, \gamma_{\mathrm{eq}}, \gamma_{R}, \boldsymbol{n}\right) \\
+ & \sum_{j=1}^{N_{s}} \frac{1}{\left|\mathcal{D}_{j}(\boldsymbol{x})\right|} \sum_{\hat{x}_{R} \in \mathcal{D}_{j}(\boldsymbol{x})} \beta Q\left(\sqrt{2 \alpha \gamma_{g_{j}}}\right) P\left(\boldsymbol{x} \rightarrow \tilde{\boldsymbol{x}} \mid \hat{x}_{R}, \boldsymbol{\gamma}_{f}, \gamma_{\mathrm{eq}}, \gamma_{R}, \boldsymbol{n}\right),
\end{aligned}
$$

where

$$
\mathcal{D}_{j}(\boldsymbol{x}) \triangleq\left\{\mu_{\mathcal{X}}\left(\mu_{\mathcal{X}}^{-1}\left(\bar{x}_{1}\right) \oplus \cdots \oplus \mu_{\mathcal{X}}^{-1}\left(\bar{x}_{N_{s}}\right)\right) \mid \bar{x}_{\nu} \in \mathcal{N}\left(x_{\nu}\right), \nu=j, \bar{x}_{\nu}=x_{\nu}, \nu \neq j\right\} .
$$

Here, for a given transmit vector $\boldsymbol{x}$, set $\mathcal{D}_{j}(\boldsymbol{x})$ collects all possible values for $\hat{x}_{R}$ assuming that $x_{j}$ is received in error at the relay, while all $x_{i}, i \neq j$, are correctly received. Furthermore, the conditional PEP $P\left(\boldsymbol{x} \rightarrow \tilde{\boldsymbol{x}} \mid \bar{x}_{R}, \gamma_{f}, \gamma_{\text {eq }}, \gamma_{R}, \boldsymbol{n}\right), \bar{x}_{R} \in\left\{x_{R}, \hat{x}_{R}\right\}$, can be written as

$$
\begin{aligned}
P\left(\boldsymbol{x} \rightarrow \tilde{\boldsymbol{x}} \mid \bar{x}_{R}, \boldsymbol{\gamma}_{f}, \gamma_{\mathrm{eq}}, \gamma_{R}, \boldsymbol{n}\right) & \triangleq \operatorname{Pr}\left\{m_{c}(\boldsymbol{x})>m_{c}(\tilde{\boldsymbol{x}}) \mid \bar{x}_{R}, \gamma_{f}, \gamma_{\mathrm{eq}}, \gamma_{R}, \boldsymbol{n}\right\} \\
& =\operatorname{Pr}\left\{\sum_{i=1}^{N_{s}} \Delta_{f_{i}}\left(x_{i}, \tilde{x}_{i}\right)+\lambda_{R} \Delta_{R}\left(x_{R}, \tilde{x}_{R}, \bar{x}_{R}\right)<0 \mid \boldsymbol{\gamma}_{f}, \gamma_{\mathrm{eq}}, \gamma_{R}, \boldsymbol{n}\right\},
\end{aligned}
$$

where

$$
\Delta_{f_{i}}\left(x_{i}, \tilde{x}_{i}\right) \triangleq\left|\sqrt{\gamma_{f_{i}}}\left(\tilde{x}_{i}-x_{i}\right)+\bar{n}_{D, i}\right|^{2}-\left|\bar{n}_{D, i}\right|^{2}
$$

and

$$
\Delta_{R}\left(x_{R}, \tilde{x}_{R}, \bar{x}_{R}\right) \triangleq\left|\sqrt{\gamma_{R}}\left(\tilde{x}_{R}-\bar{x}_{R}\right)+\bar{n}_{D, R}\right|^{2}-\left|\sqrt{\gamma_{R}}\left(x_{R}-\bar{x}_{R}\right)+\bar{n}_{D, R}\right|^{2} .
$$

For derivation of the unconditional PEP, we exploit the relations $\operatorname{Pr}\{\Delta<0\}=\frac{1}{2 \pi j} \int_{c-j \infty}^{c+j \infty} \Phi_{\Delta}(s) \frac{\mathrm{d} s}{s}$, which is valid for any random variable $\Delta$ with moment generating function (MGF) $\Phi_{\Delta}(s) \triangleq \mathcal{E}_{\Delta}\left\{\mathrm{e}^{-\Delta s}\right\}$, and $\operatorname{Pr}\left\{\hat{x}_{R}=x_{R}\right\}=1-P_{e, \text { eq }}\left(\gamma_{g}\right)=1-\beta Q\left(\sqrt{2 \alpha \gamma_{\mathrm{eq}}}\right)$, which follows from Subsection 2.3. Using these relations, we obtain the unconditional PEP from (8) and (10) as

$$
P(\boldsymbol{x} \rightarrow \tilde{\boldsymbol{x}})=\mathcal{E}_{\boldsymbol{\gamma}_{f}, \gamma_{g}, \gamma_{R}, \boldsymbol{n}}\left\{P\left(\boldsymbol{x} \rightarrow \tilde{\boldsymbol{x}} \mid \boldsymbol{\gamma}_{f}, \boldsymbol{\gamma}_{g}, \gamma_{R}, \boldsymbol{n}\right)\right\}=\frac{1}{2 \pi j} \int_{c-j \infty}^{c+j \infty}\left(\prod_{i=1}^{N_{s}} \Phi_{f_{i}}(s)\right) \Phi_{R}(s) \frac{\mathrm{d} s}{s}
$$


where $c$ is a small positive constant that lies in the region of convergence of the integrand and

$$
\begin{aligned}
& \Phi_{f_{i}}(s) \triangleq \mathcal{E}_{\gamma_{f_{i}}, \bar{n}_{D, i}}\left\{\mathrm{e}^{-s \Delta_{f_{i}}\left(x_{i}, \tilde{x}_{i}\right)}\right\}, \\
& \Phi_{R}(s) \triangleq \Phi_{R}^{c}(s)+\sum_{j=1}^{N_{s}} \frac{1}{\left|\mathcal{D}_{j}(\boldsymbol{x})\right|} \sum_{\hat{x}_{R} \in \mathcal{D}_{j}(\boldsymbol{x})} \Phi_{R, j}^{e}\left(\hat{x}_{R} ; s\right),
\end{aligned}
$$

with $\Phi_{R, j}^{e}\left(\hat{x}_{R} ; s\right)$ and $\Phi_{R}^{c}(s)$ as defined in the Appendix in Lemmas 2 and 4, respectively. Based on (13) and (15) the PEP can be expressed as

$$
P(\boldsymbol{x} \rightarrow \tilde{\boldsymbol{x}})=P_{c}(\boldsymbol{x}, \tilde{\boldsymbol{x}})+\sum_{j=1}^{N_{s}} \frac{1}{\left|\mathcal{D}_{j}(\boldsymbol{x})\right|} \sum_{\hat{x}_{R} \in \mathcal{D}_{j}(\boldsymbol{x})} P_{e, j}\left(\boldsymbol{x}, \tilde{\boldsymbol{x}}, \hat{x}_{R}\right)
$$

where

$$
P_{c}(\boldsymbol{x}, \tilde{\boldsymbol{x}}) \triangleq \frac{1}{2 \pi j} \int_{c-j \infty}^{c+j \infty}\left(\prod_{i=1}^{N_{s}} \Phi_{f_{i}}(s)\right) \Phi_{R}^{c}(s) \frac{\mathrm{d} s}{s},
$$

and

$$
P_{e, j}\left(\boldsymbol{x}, \tilde{\boldsymbol{x}}, \hat{x}_{R}\right) \triangleq \frac{1}{2 \pi j} \int_{c-j \infty}^{c+j \infty}\left(\prod_{i=1}^{N_{s}} \Phi_{f_{i}}(s)\right) \Phi_{R, j}^{e}\left(\hat{x}_{R} ; s\right) \frac{\mathrm{d} s}{s} .
$$

To facilitate the calculation of the asymptotic PEP, we now present the following proposition which sheds some light on the asymptotic behavior of the PEP $P(\boldsymbol{x} \rightarrow \tilde{\boldsymbol{x}})$.

Proposition 1: Assume without loss of generality that $\bar{\gamma}_{f_{i}}=\zeta_{f_{i}} \bar{\gamma}, \bar{\gamma}_{g_{i}}=\zeta_{g_{i}} \bar{\gamma}, 1 \leq i \leq N_{s}$, and $\bar{\gamma}_{R}=\zeta_{R} \bar{\gamma}$, where $\zeta_{f_{i}}, \zeta_{g_{i}}$ and $\zeta_{R}$ are finite (positive) constants, which are independent of $\bar{\gamma}$, and define the diversity gain associated with the PEP as $G_{d, \mathrm{PEP}} \triangleq-\lim _{\bar{\gamma} \rightarrow \infty} \log (P(\boldsymbol{x} \rightarrow \tilde{\boldsymbol{x}})) / \log (\bar{\gamma})$. The diversity gain is then given by $G_{d, \mathrm{PEP}}=d_{H}(\boldsymbol{x}, \tilde{\boldsymbol{x}})$, where $d_{H}(\boldsymbol{x}, \tilde{\boldsymbol{x}})$ denotes the Hamming distance between data vector $\boldsymbol{s}_{e}$ and $\tilde{\boldsymbol{s}}_{e}=\left[\tilde{s}_{1}, \cdots, \tilde{s}_{N_{s}}, \tilde{s}_{R}\right]^{T} \in \mathcal{A}^{N_{s}+1}$. Furthermore, for all possible pairs $(\boldsymbol{x}, \tilde{\boldsymbol{x}})$ we have $d_{H}(\boldsymbol{x}, \tilde{\boldsymbol{x}}) \geq 2$.

Please refer to the Appendix for a proof of Proposition 1. From Proposition 1 we conclude that for calculation of the asymptotic SER based on (5), only error events with $d_{H}(\boldsymbol{x}, \tilde{\boldsymbol{x}})=2$ have to be included since error events with $d_{H}(\boldsymbol{x}, \tilde{\boldsymbol{x}})>2$ yield a higher diversity gain and thus, their contribution to the asymptotic SER is negligible. Therefore, in the following, we calculate the asymptotic PEP only for error events with $d_{H}(\boldsymbol{x}, \tilde{\boldsymbol{x}})=2$. For clarity, we consider the cases $x_{R} \neq \tilde{x}_{R}$ and $x_{R}=\tilde{x}_{R}$ separately.

Case $1\left(\boldsymbol{x}_{\boldsymbol{R}} \neq \tilde{\boldsymbol{x}}_{\boldsymbol{R}}\right)$ : It is easy to see that in this case, $d_{j} \triangleq\left|x_{j}-\tilde{x}_{j}\right|, 1 \leq j \leq N_{s}$, is non-zero only for a single value of index $j$, i.e., we have $d_{j} \neq 0, j=i$, and $d_{j}=0, j \neq i$. As a result, from Lemma 1 we obtain $\Phi_{f_{j}}(s) \stackrel{\circ}{=} \frac{1}{d_{j}^{2} s(1-s) \bar{\gamma}_{f_{j}}}, j=i$ and $\Phi_{f_{j}}(s) \stackrel{\circ}{=} 1, j \neq i$. Therefore, using (17) and Lemma 4 
we arrive at

$$
P_{c}(\boldsymbol{x}, \tilde{\boldsymbol{x}}) \stackrel{\circ}{=} \frac{1}{2 \pi^{2} j} \int_{0}^{\pi / 2} \int_{c-j \infty}^{c+j \infty} \frac{1}{d_{i}^{2} s(1-s) \bar{\gamma}_{f_{i}}}\left(\frac{2}{\bar{\gamma}_{\mathrm{eq}} d_{R}^{2} s}+\frac{2}{\bar{\gamma}_{R} d_{R}^{2} s(1-s)}-\frac{\beta}{\bar{\gamma}_{\mathrm{eq}} d_{R}^{2}\left(s+\frac{\alpha}{\sin ^{2} \theta d_{R}^{2}}\right)}\right) \frac{\mathrm{d} s}{s} \mathrm{~d} \theta,
$$

where $d_{R} \triangleq\left|x_{R}-\tilde{x}_{R}\right|$. The inner complex integral in (19) can be calculated using standard inverse Laplace transform techniques such as partial fraction expansion. This leads to

$$
P_{c}(\boldsymbol{x}, \tilde{\boldsymbol{x}}) \stackrel{\circ}{=} \frac{1}{\bar{\gamma}_{f_{i}}}\left(\phi_{c}^{g}(\boldsymbol{x}, \tilde{\boldsymbol{x}}) \sum_{i=1}^{N_{s}} \frac{1}{\bar{\gamma}_{g_{i}}}+\frac{\phi_{c}^{R}(\boldsymbol{x}, \tilde{\boldsymbol{x}})}{\bar{\gamma}_{R}}\right)
$$

where

$$
\phi_{c}^{g}(\boldsymbol{x}, \tilde{\boldsymbol{x}}) \triangleq \frac{2-\beta+\frac{\beta \alpha}{\sqrt{\alpha^{2}+\alpha d_{R}^{2}}}}{2 d_{i}^{2} d_{R}^{2}} \text { and } \phi_{c}^{R}(\boldsymbol{x}, \tilde{\boldsymbol{x}}) \triangleq \frac{3}{d_{i}^{2} d_{R}^{2}} .
$$

Furthermore, from (18) and Lemma 2 we have

$$
P_{e, j}\left(\boldsymbol{x}, \tilde{\boldsymbol{x}}, \hat{x}_{R}\right) \stackrel{\circ}{=} \frac{\beta}{2 \pi^{2} j} \int_{0}^{\pi / 2} \int_{c-j \infty}^{c+j \infty} \frac{1}{d_{i}^{2} s^{2}(1-s)\left(\bar{d}_{R}\left(\hat{x}_{R}\right) s+\frac{\alpha}{\sin ^{2} \theta}\right) \bar{\gamma}_{f_{i}} \bar{\gamma}_{g_{j}}} \mathrm{~d} s \mathrm{~d} \theta=\frac{\phi_{e}\left(\boldsymbol{x}, \tilde{\boldsymbol{x}}, \hat{x}_{R}\right)}{\bar{\gamma}_{f_{i}} \bar{\gamma}_{g_{j}}}
$$

with

$$
\phi_{e}\left(\boldsymbol{x}, \tilde{\boldsymbol{x}}, \hat{x}_{R}\right)= \begin{cases}\frac{\beta}{2 d_{i}^{2} d_{R}\left(\hat{x}_{R}\right)}-\frac{\beta \alpha}{2 d_{i}^{2} \bar{d}_{R}\left(\hat{x}_{R}\right) \sqrt{\alpha^{2}+\alpha \bar{d}_{R}\left(\hat{x}_{R}\right)}} & \bar{d}_{R}\left(\hat{x}_{R}\right)>0 \\ \frac{\beta}{4 \alpha d_{i}^{2}}-\frac{3 \beta \bar{d}_{R}\left(\hat{x}_{R}\right)}{16 d_{i}^{2} \alpha^{2}} & \bar{d}_{R}\left(\hat{x}_{R}\right) \leq 0\end{cases}
$$

where $\bar{d}_{R}\left(\hat{x}_{R}\right) \triangleq\left|\tilde{x}_{R}-\hat{x}_{R}\right|^{2}-\left|x_{R}-\hat{x}_{R}\right|^{2}$.

Case $2\left(\boldsymbol{x}_{\boldsymbol{R}}=\tilde{\boldsymbol{x}}_{\boldsymbol{R}}\right)$ : In this case, $d_{j}$ is non-zero for two values of index $j$, i.e., we have $d_{j} \neq 0$, $j \in\left\{i_{1}, i_{2}\right\}$, and $d_{j}=0$, otherwise. Thus, based on Lemma 1 , we obtain $\Phi_{f_{j}}(s) \stackrel{\circ}{=} \frac{1}{d_{j}^{2} s(1-s) \bar{\gamma}_{f_{j}}}$, $j \in\left\{i_{1}, i_{2}\right\}$, and $\Phi_{f_{j}}(s) \stackrel{\circ}{=}$, otherwise. Furthermore, in this case, $d_{R}=0$ is valid, and therefore, based on Lemma 4 , we obtain $\Phi_{R}^{c}(s) \stackrel{\circ}{=}$. Thus, using (17) we obtain

$$
P_{c}(\boldsymbol{x}, \tilde{\boldsymbol{x}}) \doteq \frac{1}{2 \pi^{2} j} \int_{0}^{\pi / 2} \int_{c-j \infty}^{c+j \infty} \frac{1}{d_{i_{1}}^{2} d_{i_{2}}^{2} s^{3}(1-s)^{2} \bar{\gamma}_{f_{i_{1}}} \bar{\gamma}_{f_{i_{2}}}} \mathrm{~d} s \mathrm{~d} \theta=\frac{\bar{\phi}_{c}(\boldsymbol{x}, \tilde{\boldsymbol{x}})}{\bar{\gamma}_{f_{i_{1}}} \bar{\gamma}_{f_{i_{2}}}}
$$

with $\bar{\phi}_{c}(\boldsymbol{x}, \tilde{\boldsymbol{x}}) \triangleq \frac{3}{d_{i_{1}}^{2} d_{i_{2}}^{2}}$. Furthermore, from (18) and Lemma 2 we get

$$
P_{e, j}\left(\boldsymbol{x}, \tilde{\boldsymbol{x}}, \hat{x}_{R}\right) \stackrel{1}{=} \frac{1}{2 \pi^{2} j} \int_{0}^{\pi / 2} \int_{c-j \infty}^{c+j \infty} \frac{\beta}{d_{i_{1}}^{2} d_{i_{2}}^{2} s^{3}(1-s)^{2}\left(\bar{d}_{R}\left(\hat{x}_{R}\right) s+\frac{\alpha}{\sin ^{2} \theta}\right) \bar{\gamma}_{f_{i_{1}}} \bar{\gamma}_{f_{i_{2}}} \bar{\gamma}_{g_{j}}} \mathrm{~d} s \mathrm{~d} \theta=\frac{\bar{\phi}{ }_{e}\left(\boldsymbol{x}, \tilde{\boldsymbol{x}}, \hat{x}_{R}\right)}{\bar{\gamma}_{f_{i_{1}}} \bar{\gamma}_{f_{i_{2}}} \bar{\gamma}_{g_{j}}}
$$

where $\bar{\phi}_{e}\left(\boldsymbol{x}, \tilde{\boldsymbol{x}}, \hat{x}_{R}\right)$ is a (positive) finite constant which does not appear in the final SER and BER expressions.

With these asymptotic expressions for $P_{c}(\boldsymbol{x}, \tilde{\boldsymbol{x}})$ and $P_{e, j}\left(\boldsymbol{x}, \tilde{\boldsymbol{x}}, \hat{x}_{R}\right)$ at hand, a closed-form expression for the asymptotic PEP can be calculated based on (16). 


\subsection{Asymptotic SER and BER}

In order to obtain an accurate expression for the asymptotic SER, we first expurgate the union bound in (5) according to Proposition 1. In particular, we only include error events with $d_{H}(\boldsymbol{x}, \tilde{\boldsymbol{x}})=2$ in the union bound since the contribution of error events with $d_{H}(\boldsymbol{x}, \tilde{\boldsymbol{x}})>2$ to the asymptotic SER is negligible (cf. Proposition 1). This expurgation is accomplished by replacing the set $\mathcal{B}_{i}(\boldsymbol{x})$ in (6) with subset

$$
\mathcal{C}_{i}(\boldsymbol{x}) \triangleq\left\{\tilde{\boldsymbol{x}} \mid \tilde{x}_{j} \in \mathcal{X} \backslash\left\{x_{j}\right\}, j=i, \tilde{x}_{j} \in \mathcal{X}, j \neq i, d_{H}(\boldsymbol{x}, \tilde{\boldsymbol{x}})=2\right\} .
$$

We are now ready to state our main result. In particular, in the following proposition, we combine (5), (16), and (26) to obtain a general and accurate expression for the asymptotic SER which is valid for arbitrary numbers of sources, arbitrary signal constellations, and arbitrary constellation mappings (refer to the Appendix for a proof).

Proposition 2: For the NCCD system described in Section 2, an accurate expression for the asymptotic SER of the $i$ th source can be obtained $\mathrm{as}^{2}$

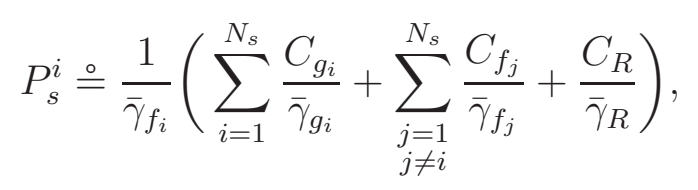

where

$$
\begin{aligned}
& C_{g_{i}} \triangleq \frac{1}{M^{N_{s}}} \sum_{\boldsymbol{x} \in \mathcal{X}^{N_{s}}} \sum_{\tilde{\boldsymbol{x}} \in \mathcal{C}_{i}^{i}(\boldsymbol{x})}\left(\phi_{c}^{g}(\boldsymbol{x}, \tilde{\boldsymbol{x}})+\frac{1}{\left|\mathcal{D}_{j}(\boldsymbol{x})\right|} \sum_{\hat{x}_{R} \in \mathcal{D}_{j}(\boldsymbol{x})} \phi_{e}\left(\boldsymbol{x}, \tilde{\boldsymbol{x}}, \hat{x}_{R}\right)\right), \\
& C_{f_{j}} \triangleq \frac{1}{M^{N_{s}}} \sum_{\boldsymbol{x} \in \mathcal{X}^{N_{s}}} \sum_{\tilde{\boldsymbol{x}} \in \mathcal{C}_{i}^{j}(\boldsymbol{x})} \bar{\phi}_{c}(\boldsymbol{x}, \tilde{\boldsymbol{x}}), \quad \text { and } \quad C_{R} \triangleq \frac{1}{M^{N_{s}}} \sum_{\boldsymbol{x} \in \mathcal{X}^{N_{s}}} \sum_{\tilde{\boldsymbol{x}} \in \mathcal{C}_{i}^{i}(\boldsymbol{x})} \phi_{c}^{R}(\boldsymbol{x}, \tilde{\boldsymbol{x}}) .
\end{aligned}
$$

In (28) and (29), $\mathcal{C}_{i}^{l}(\boldsymbol{x}), 1 \leq l \leq N_{s}$, is defined as

$$
\mathcal{C}_{i}^{l}(\boldsymbol{x}) \triangleq\left\{\tilde{\boldsymbol{x}} \mid \tilde{x}_{j} \neq x_{j}, j \in\{i, l\}, \tilde{x}_{j}=x_{j}, \text { otherwise, } d_{H}(\boldsymbol{x}, \tilde{\boldsymbol{x}})=2\right\} .
$$

Remark 2: The asymptotic SER in (27) is, in general, a function of the constellation mapping $\mu_{\mathcal{X}}$ because the sets $\mathcal{C}_{i}^{l}(\boldsymbol{x})$ and $\mathcal{D}_{j}(\boldsymbol{x})$ and consequently the coefficients $C_{g_{j}}, C_{f_{j}}$, and $C_{R}$ depend on the constellation mapping. We will study this dependency in Section 5 where we show that some performance improvement can be achieved by optimizing the mapping $\mu_{\mathcal{X}}$. In case of a BPSK constellation, however, the two possible mappings are equivalent and lead to the same expression for the asymptotic SER. Specifically, based on (27) the asymptotic BER of BPSK (which is identical to

\footnotetext{
${ }^{2}$ For BPSK modulation, the SER expression in (27) is asymptotically exact. A comparison with simulations suggests that for general $M$-ary modulation, the SER in (27) is a tight upper bound, cf. Section 5.
} 
the asymptotic SER) is obtained as

$$
P_{b, \mathrm{BPSK}}^{i} \stackrel{\circ}{=} \frac{1}{\bar{\gamma}_{f_{i}}}\left(C_{\mathrm{BPSK}}^{1} \sum_{i=1}^{N_{s}} \frac{1}{\bar{\gamma}_{g_{i}}}+C_{\mathrm{BPSK}}^{2}\left[\sum_{\substack{j=1 \\ j \neq i}}^{N_{s}} \frac{1}{\bar{\gamma}_{f_{j}}}+\frac{1}{\bar{\gamma}_{R}}\right]\right)
$$

where $C_{\mathrm{BPSK}}^{1} \triangleq \frac{45+\sqrt{5}}{160}$ and $C_{\mathrm{BPSK}}^{2} \triangleq \frac{3}{16}$.

Remark 3: Letting $\bar{\gamma}_{f_{i}}=\zeta_{f_{i}} \bar{\gamma}, \bar{\gamma}_{g_{i}}=\zeta_{g_{i}} \bar{\gamma}, 1 \leq i \leq N_{s}$, and $\bar{\gamma}_{R}=\zeta_{R} \bar{\gamma}$, where $\zeta_{f_{i}}, \zeta_{g_{i}}$, and $\zeta_{R}$ are finite

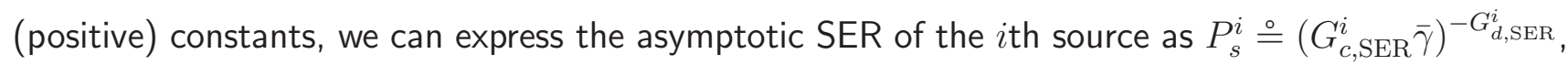
where $G_{d, \mathrm{SER}}^{i}$ and $G_{c, \mathrm{SER}}^{i}$ are the SER-based diversity gain and network-coding gain, respectively. Thus, $G_{d, \mathrm{SER}}^{i}$ and $G_{c, \mathrm{SER}}^{i}$ correspond to the negative asymptotic slope and a relative horizontal shift of the SER curve when plotted as a function of $\bar{\gamma}$ on a double-logarithmic scale, respectively. Based on (27) we therefore obtain

$$
G_{d, \mathrm{SER}}^{i}=2, \quad G_{c, \mathrm{SER}}^{i}[\mathrm{~dB}]=5 \log _{10}\left(\zeta_{f_{i}}\right)-5 \log _{10}\left(\sum_{i=1}^{N_{s}} \frac{C_{g_{i}}}{\zeta_{g_{i}}}+\sum_{\substack{j=1 \\ j \neq i}}^{N_{s}} \frac{C_{f_{j}}}{\zeta_{f_{j}}}+\frac{C_{R}}{\zeta_{R}}\right) .
$$

From (32) it is evident that $G_{d, \mathrm{SER}}^{i}=2$ is achieved irrespective of the number of sources $N_{s}$. Furthermore, for the network-coding gain, $G_{c, \mathrm{SER}}^{i}$, we make the following observations. $G_{c, \mathrm{SER}}^{i}$ is a function of the number of sources $N_{s}$, the signal constellation $\mathcal{X}$, the constellation mapping $\mu_{\mathcal{X}}$, as well as the relative link qualities $\zeta_{f_{i}}, \zeta_{g_{i}}$, and $\zeta_{R}$. Eq. (32) reveals that for $\zeta_{f_{i}}=\zeta_{g_{i}}=\zeta_{R}$, the networkcoding gain increases only logarithmically with increasing $N_{s}$. Furthermore, for NCCD systems where the $R \rightarrow D$ link is the bottleneck link, i.e., $\zeta_{R} \ll \zeta_{f_{i}}, \zeta_{g_{i}}, 1 \leq i \leq N_{s}, G_{c, \text { SER }}^{i}$ can be approximated as $G_{c, \mathrm{SER}}^{i} \approx 5 \log _{10}\left(\zeta_{f_{i}} \zeta_{R} / C_{R}\right)$, implying that the network-coding gain is practically independent of the number of sources. The above observations will be confirmed in Section 5 with simulation results.

Remark 4: Having obtained the asymptotic SER from (27), for Gray labeling, the asymptotic BER of the $i$ th source, $P_{b}^{i}$, can be tightly approximated as $P_{b}^{i} \stackrel{\circ}{=} \frac{1}{\log _{2}(M)} P_{s}^{i}$.

\section{Optimization of NCCD Systems}

In addition to the mapping optimization discussed in the previous section, the obtained analytical error rate expressions can be exploited to formulate various practically relevant optimization problems for NCCD systems. In particular, as was done in $[14,15]$ for conventional amplify-and-forward CD, we may formulate optimal power allocation (OPA), relay selection, and relay placement problems. Due to space limitations, we concentrate in the following on OPA. In particular, in this section, we investigate the optimal allocation of the source and relay powers, $P_{i}, 1 \leq i \leq N_{s}$, and $P_{R}$ in NCCD systems for a given power budget. 
Based on the asymptotic SER given in (27), the OPA optimization problem can be mathematically cast as

$$
\begin{aligned}
\min _{P_{1}, \ldots, P_{N_{s}}, P_{R}} & \sum_{i=1}^{N_{s}} \psi_{i}\left(\frac{1}{P_{i} \xi_{f_{i}}}\left[\sum_{i=1}^{N_{s}} \frac{C_{g_{i}}}{P_{i} \xi_{g_{i}}}+\sum_{\substack{j=1 \\
j \neq i}}^{N_{s}} \frac{C_{f_{j}}}{P_{j} \xi_{f_{j}}}+\frac{C_{R}}{P_{R} \xi_{R}}\right]\right) \\
\text { subject to : } \quad & \sum_{i=1}^{N_{s}} P_{i}+P_{R} \leq P_{t} \\
& 0 \leq P_{i} \leq P_{i, \max }, \quad 1 \leq i \leq N_{s} \\
& 0 \leq P_{R} \leq P_{R, \max },
\end{aligned}
$$

where $\psi_{i}(\cdot)$ is an increasing convex cost function which can be chosen to achieve certain design goals, $P_{t}$ is the total power budget, $P_{i, \max }$ and $P_{R, \max }$ denote the maximum power available at the $i$ th source and the relay, respectively, and we have defined the link statistics $\xi_{f_{i}} \triangleq \Omega_{f_{i}} / \sigma_{n_{D, i}}^{2}, \xi_{g_{i}} \triangleq \Omega_{g_{i}} / \sigma_{n_{R, i}}^{2}$, and $\xi_{R} \triangleq \Omega_{R} / \sigma_{n_{R}}^{2}$, respectively.

It is easy to see that the solution set of the linear constraints (33b)-(33d) is non-empty, and therefore the optimization problem is always feasible. Furthermore, using the transformation of variables $P_{i}=\log \left(\tilde{P}_{i}\right), 1 \leq i \leq N_{s}$, and $P_{R}=\log \left(\tilde{P}_{R}\right)$ optimization problem (33) is transformed into a convex optimization problem in the new variables $\tilde{P}_{i}$ and $\tilde{P}_{R}$. The resulting convex problem can be efficiently solved using well-known interior point methods [16]. We note that as is customary in the literature, we assume that the OPA is computed at the destination terminal, which subsequently informs the sources and the relay of their assigned transmission power via a low-rate feedback channel. To compute the OPA the destination requires knowledge about the channel statistics $\xi_{f_{i}}, \xi_{g_{i}}$, $1 \leq i \leq N_{s}$, and $\xi_{R}$. The destination can estimate $\xi_{f_{i}}, 1 \leq i \leq N_{s}$, and $\xi_{R}$, directly as the required information is readily available at the destination. $\xi_{g_{i}}, 1 \leq i \leq N_{s}$, can be estimated at the relay and then fed back to the destination via another low-rate feedback channel.

For cost function $\psi_{i}(\cdot)$, the two special cases, $\psi_{i}(x)=x$ and $\psi_{i}(x)=\exp (\rho x), \rho \rightarrow \infty$, are of particular interest which lead to a minimum average SER and a min-max fair design, respectively. For the purpose of OPA in NCCD systems the latter appears to be practically more appealing since minimizing the average SER may favor sources with good link qualities and result in solutions that are unfair to the other sources [17]. Therefore, in the following, we focus on the min-max fair design which aims at minimizing the maximum SER among all sources. In particular, letting $\psi_{i}(x)=\exp (\rho x)$, $\rho \rightarrow \infty$, in (33) the power allocation problem can be equivalently stated as

$$
\min _{P_{1}, \ldots, P_{N_{s}}, P_{R}} \quad \max _{i}\left\{\frac{1}{P_{i} \xi_{f_{i}}}\left[\sum_{i=1}^{N_{s}} \frac{C_{g_{i}}}{P_{i} \xi_{g_{i}}}+\sum_{\substack{j=1 \\ j \neq i}}^{N_{s}} \frac{C_{f_{j}}}{P_{j} \xi_{f_{j}}}+\frac{C_{R}}{P_{R} \xi_{R}}\right]\right\}
$$

subject to : Constraints $(33 \mathrm{~b})-(33 \mathrm{~d})$. 
Introducing an auxiliary variable $\nu$, problem (34) can be further transformed into

$$
\begin{array}{ll}
\min _{P_{1}, \ldots, P_{N_{s}}, P_{R}, \nu \geq 0} & \nu \\
\text { subject to : } & \frac{1}{P_{i} \xi_{f_{i}}}\left[\sum_{i=1}^{N_{s}} \frac{C_{g_{i}}}{P_{i} \xi_{g_{i}}}+\sum_{\substack{j=1 \\
j \neq i}}^{N_{s}} \frac{C_{f_{j}}}{P_{j} \xi_{f_{j}}}+\frac{C_{R}}{P_{R} \xi_{R}}\right] \leq \nu, \quad 1 \leq i \leq N_{s} \\
& \text { Constraints }(33 \mathrm{~b})-(33 \mathrm{~d}) .
\end{array}
$$

Since both the objective function and constraints can be written in the form of posynomials, optimization problem (35) is a geometric program (GP) which can be efficiently solved using standard tools from the literature $[16,17]$.

\section{Results and System Optimization}

In this section, we use the derived the analytical results to investigate the impact of the various system and channel parameters on the performance of NCCD systems and to optimize the performance of these systems. For all figures shown this section, the asymptotic BER of BPSK and the asymptotic SER of higher order modulation schemes were obtained based on (31) and (27), respectively. Unless specified otherwise, we assume generalized C-MRC detection at the destination.

\subsection{Performance of NCCD Systems}

In Fig. 2, we show the BER of an NCCD system with $N_{s}=2$ sources and BPSK modulation for the generalized C-MRC detection scheme as well as ML detection. We assume $\bar{\gamma}_{f_{1}}=\bar{\gamma}_{f_{2}} \triangleq \bar{\gamma}_{f}$ and $\bar{\gamma}_{g_{1}}=\bar{\gamma}_{g_{2}} \triangleq \bar{\gamma}_{g}$ and show results for four combinations of the channel quality vector $\left(\bar{\gamma}_{f}, \bar{\gamma}_{g}, \bar{\gamma}_{R}\right)$. We note that due to the symmetry of the network, the BERs of both sources are identical. For C-MRC detection the the analytical results (dashed lines) are in excellent agreement with the corresponding simulation results (solid lines with markers) for sufficiently high SNR, which confirms the accuracy of the approximations made in Sections 2 and 3. Furthermore, the simulated BER results for ML combining at the destination (dash-dotted lines) are practically identical to the BERs achieved with generalized C-MRC, which confirms the viability of generalized C-MRC. We also observe from Fig. 2 that, as expected from the analysis in Section 3 (cf. Remark 3), the network-coding gain is a function of the respective channels qualities but the diversity gain is equal to two for all channel quality settings. Furthermore, having a relatively strong $S \rightarrow D$ channel is most beneficial in terms of BER performance. However, this scenario may not be realistic in practice since the relay is usually closer to the sources than the destination.

In Fig. 3, we investigate the BER of an asymmetric NCCD system with $N_{s}=4$ sources and BPSK modulation. For this system, we have assumed $\bar{\gamma}_{f_{1}} \triangleq \bar{\gamma}, \bar{\gamma}_{f_{2}}=\bar{\gamma}+10 \mathrm{~dB}, \bar{\gamma}_{f_{3}}=\bar{\gamma}+16 \mathrm{~dB}$, 
$\bar{\gamma}_{f_{4}}=\bar{\gamma}+20 \mathrm{~dB}$, and $\bar{\gamma}_{g_{1}}=\bar{\gamma}_{g_{2}}=\bar{\gamma}_{g_{3}}=\bar{\gamma}_{g_{4}}=\bar{\gamma}_{R}=\bar{\gamma}$. The BER of each source as well as the average BER of all sources are shown as a function of $\bar{\gamma}$ for both generalized C-MRC (simulation and asymptotic results) and ML combining. We observe that although the diversity gain for each source is equal to two, the network-coding gain is source dependent because of the non-identical channel qualities of the sources. Again, for generalized C-MRC the analytical results are in excellent agreement with the simulations at high SNRs, and the performance gain achievable with ML combining compared to generalized $\mathrm{C}-\mathrm{MRC}$ is negligible.

In Fig. 4, we study the impact of number of sources on the performance of NCCD systems. Thereby, we consider an NCCD system with BPSK modulation, $\bar{\gamma}_{f_{i}}=\bar{\gamma}_{g_{i}}=\bar{\gamma}, 1 \leq i \leq N_{s}$, and $\bar{\gamma}_{R}=\bar{\gamma}$ and show the average BER for different $N_{s}$ as a function of $\bar{\gamma}$ for BPSK. Asymptotic BER results are shown for three values of $\bar{\gamma}_{R}$, but corresponding simulation results are shown only for two $\bar{\gamma}_{R}$ values for clarity of presentation. As expected, a diversity gain of two is achieved in all cases irrespective of the number of sources. Furthermore, in accordance with Remark 3, we observe that for $\bar{\gamma}_{R}=\bar{\gamma}$ the network-coding gain increases only logarithmically with $N_{s}$. In addition, as $\bar{\gamma}_{R}$ decreases (i.e., the $R \rightarrow D$ link becomes the bottleneck link), the network-coding gain becomes less dependent on $N_{s}$ and is rendered practically independent of $N_{s}$ for low enough $\bar{\gamma}_{R}$. We also note that although increasing $N_{s}$ results in some BER performance degradation, in general, this loss is more than compensated by the associated gain in throughput (cf. Remark 1).

\subsection{Performance Optimization}

As discussed in Remark 2, the performance of NCCD systems with non-binary modulation can be improved by optimizing the constellation mapping $\mu_{\mathcal{X}}$. The optimal mapping depends on the qualities of the different channels. As an example, we consider two different channel quality settings for a NCCD system with $N_{s}=2$ : Case I with $\bar{\gamma}_{f_{1}}=\bar{\gamma}_{f_{2}}=\bar{\gamma}_{R}=\bar{\gamma}, \bar{\gamma}_{g_{1}}=\bar{\gamma}_{g_{2}}=\bar{\gamma}+30 \mathrm{~dB}$ and Case II with $\bar{\gamma}_{f_{1}}=\bar{\gamma}_{f_{2}}=\bar{\gamma}_{g_{1}}=\bar{\gamma}_{g_{2}}=\bar{\gamma}, \bar{\gamma}_{R}=\bar{\gamma}-30 \mathrm{~dB}$. For both cases, we performed a search over all possible constellation mappings for 8-PSK and 16-QAM modulation to find the mapping which minimizes the asymptotic SER in (27), respectively. The results for this search along with a natural mapping for both constellations are shown in Figs. 5 and 6 . We note that in both cases the optimal mapping is not unique as rotations of the mapping do not affect performance.

For the 16-QAM mappings in Fig. 6, the simulated and asymptotic SERs are shown in Fig. 7 as functions of $\bar{\gamma}$. Fig. 7 reveals that for non-binary signal constellations the agreement between simulation results and analytical results is not as good as for BPSK modulation. The reason for the discrepancy is the union bound in (5), which, despite the employed expurgation, still overestimates the SER to some extent. Nevertheless, for both considered cases the analytical SER upper bound accurately predicts the performance difference between the optimal and natural mappings, suggesting 
that this upper bound is a useful tool for optimization of the constellation mapping. As can be observed from Fig. 7, in both considered cases a performance gain of $1 \mathrm{~dB}$ is achieved by the optimal mapping compared to the natural mapping.

For the 8-PSK mappings shown in Fig. 5, the optimal mappings achieve performance gains of 0.8 $\mathrm{dB}$ compared to the natural mapping for Cases I and II. However, in the interest of space, we do not show corresponding SER results.

In Fig. 8, we consider the min-max fair OPA described in Section 4 for an NCCD system with BPSK, $N_{s}=2, \Omega_{f_{1}}=\Omega_{g_{1}}=1, \Omega_{f_{2}}=\Omega_{g_{2}}=50, \Omega_{R}=200$, and $\sigma_{n_{D, i}}^{2}=\sigma_{n_{R, i}}^{2}=\sigma_{n_{D, R}}^{2} \triangleq \sigma^{2}$. In order to investigate the maximum benefits of OPA, we omit the per-node power constraints (33c) and (33d) in (35) by letting $P_{i, \max }=\infty, i \in\{1,2\}$, and $P_{R, \max }=\infty$. The individual BERs of both sources $S_{i}, i \in\{1,2\}$ as well as the average BER of both sources are shown as functions of $P_{t} / \sigma^{2}$ for OPA $\left(P_{1}=0.87 \times P_{t}, P_{2}=0.10 \times P_{t}, P_{R}=0.03 \times P_{t}\right)$ and equal power allocation EPA $\left(P_{1}=P_{2}=P_{R}=P_{t} / 3\right)$, respectively. Since $S_{1}$ has a weaker channel, and therefore a higher BER compared to $S_{2}$, OPA aims at minimizing the BER of $S_{1}$ and improves the corresponding BER by 3.5 $\mathrm{dB}$. This performance improvement is achieved by allocating more power to $S_{1}$ compared to $S_{2}$ and the relay, and at the expense of a small degradation in the BER of $S_{2}$. However, the BER degradation suffered by $S_{2}$, if OPA is applied instead of EPA, is small compared to the gain experienced by $S_{1}$. Consequently, OPA also improves the average BER by $3.2 \mathrm{~dB}$ over EPA.

\section{Conclusions}

In this paper, we studied NCCD systems employing $\operatorname{GF}\left(2^{m}\right)$ network coding and developed a simple generalized C-MRC scheme which achieves the maximum diversity of the considered system even if erroneous decisions at the relay are taken into account. Assuming independent Rayleigh fading for all links in the network, we derived closed-form expressions for the asymptotic SER and BER of the considered NCCD system. These simple and elegant expressions provide insight into the impact of various system and channel parameters on performance and can be exploited for design and optimization of NCCD systems. Simulation results confirmed the accuracy of the presented asymptotic SER and BER results and facilitated the following insights: 1) The performance loss of generalized C-MRC compared to optimal ML combining is negligible. 2) All sources achieve a diversity gain of two irrespective of the number of sources while the source dependent network-coding gain is affected by various system and channel parameters. 3) Both constellation mapping optimization and optimal power allocation can considerably improve the performance of NCCD systems. 


\section{Appendix}

In this appendix, we provide Lemmas $1-4$ and prove Propositions 1 and 2 .

Lemma 1: The asymptotic behavior of $\Phi_{f_{i}}(s), 1 \leq i \leq N_{s}$, for $\bar{\gamma}_{f_{i}} \rightarrow \infty$ is given by

$$
\Phi_{f_{i}}(s) \stackrel{\circ}{=} \frac{1}{d_{i}^{2} s(1-s) \bar{\gamma}_{f_{i}}}
$$

for $d_{i} \triangleq\left|x_{i}-\tilde{x}_{i}\right| \neq 0$ and $\Phi_{f_{i}}(s) \stackrel{\circ}{=}$ for $d_{i}=0$.

Proof. This result can be proved following the same steps as in [18, Section IV.A]. A detailed proof is omitted here because of space limitations.

Lemma 2: The asymptotic behavior of $\Phi_{R, j}^{e}\left(\hat{x}_{R} ; s\right) \triangleq \mathcal{E}_{\gamma_{g}, \gamma_{R}, \bar{n}_{D, R}}\left\{\beta Q\left(\sqrt{2 \alpha \gamma_{g_{j}}}\right) \mathrm{e}^{-s \lambda_{R} \Delta_{R}\left(x_{R}, \tilde{x}_{R}, \hat{x}_{R}\right)}\right\}$ for $\bar{\gamma}_{g_{i}} \rightarrow \infty, 1 \leq i \leq N_{s}, \bar{\gamma}_{R} \rightarrow \infty$ is given by

$$
\Phi_{R, j}^{e}\left(\hat{x}_{R} ; s\right) \stackrel{\circ}{=} \int_{0}^{\pi / 2} \frac{\beta}{\bar{\gamma}_{g_{j}}\left(\bar{d}_{R}\left(\hat{x}_{R}\right) s+\frac{\alpha}{\sin ^{2} \theta}\right)} \mathrm{d} \theta,
$$

where $\bar{d}_{R}\left(\hat{x}_{R}\right) \triangleq\left|\tilde{x}_{R}-\hat{x}_{R}\right|^{2}-\left|x_{R}-\hat{x}_{R}\right|^{2}$.

Proof. Using the alternative representation of the Q-function, $Q(x)=\frac{1}{\pi} \int_{0}^{\pi / 2} \mathrm{e}^{-x^{2} / \sin ^{2} \theta} \mathrm{d} \theta$, we can write

$$
\Phi_{R, j}^{e}\left(\hat{x}_{R} ; s\right)=\frac{\beta}{\pi} \int_{0}^{\pi / 2} \mathcal{E}_{\bar{n}_{D, R}}\{\Phi(s, \theta)\} \mathrm{d} \theta
$$

where $\Phi(s, \theta) \triangleq \mathcal{E}_{\gamma_{g}, \gamma_{R}}\left\{\mathrm{e}^{-\frac{\alpha \gamma_{g_{j}}}{\sin ^{2} \theta}} \mathrm{e}^{-s \lambda_{R} \Delta_{R}\left(x_{R}, \tilde{x}_{R}, \hat{x}_{R}\right)}\right\}$. Furthermore, from (12) we have

$$
\lambda_{R} \Delta_{R}\left(x_{R}, \tilde{x}_{R}, \hat{x}_{R}\right)=\gamma_{m} \bar{d}_{R}\left(\hat{x}_{R}\right)+\frac{2 \gamma_{m}}{\sqrt{\gamma_{R}}} d_{R} \Re\left\{\bar{n}_{D, R}^{*}\right\},
$$

with $\gamma_{m} \triangleq \min \left\{\gamma_{\mathrm{eq}}, \gamma_{R}\right\}$. Using the Taylor series expansion $\mathrm{e}^{x}=\sum_{i=0}^{\infty} x^{i} / i$ ! leads to

$$
\Phi(s, \theta)=\sum_{i=0}^{\infty} \frac{2^{i} \eta_{i}}{(2 i) !}\left|\bar{n}_{D, R}\right|^{2 i} s^{2 i} \Psi_{i}(s, \theta),
$$

with $\eta_{i} \triangleq \frac{\Gamma(i+1 / 2)}{\sqrt{\pi} \Gamma(i+1)}$ and

$$
\begin{aligned}
& \Psi_{i}(s, \theta) \triangleq \mathcal{E}_{\gamma_{g}, \gamma_{R}}\left\{\mathrm{e}^{-\left(\gamma_{m} \bar{d}_{R}\left(\hat{x}_{R}\right) s+\frac{\alpha \gamma_{g_{j}}}{\sin ^{2} \theta}\right)}\left(\frac{\gamma_{m} d_{R}}{\sqrt{\gamma_{R}}}\right)^{2 i}\right\} \\
& \quad=\frac{d_{R}^{2 i}}{\bar{\gamma}_{g_{j}} \bar{\gamma}_{R} \bar{\gamma}_{u}} \int_{0}^{\infty} \int_{0}^{\infty} \int_{0}^{\infty} \mathrm{e}^{-\left(\gamma_{m} \bar{d}_{R}\left(\hat{x}_{R}\right) s+\frac{\alpha \gamma_{g_{j}}}{\sin ^{2} \theta}\right)} \gamma_{m}^{2 i} \gamma_{R}^{-i} \mathrm{e}^{-\gamma_{g_{j}} / \bar{\gamma}_{g_{j}}} \mathrm{e}^{-\gamma_{R} / \bar{\gamma}_{R}} \mathrm{e}^{-\gamma_{u} / \bar{\gamma}_{u}} \mathrm{~d} \gamma_{g_{j}} \mathrm{~d} \gamma_{R} \mathrm{~d} \gamma_{u} .
\end{aligned}
$$

The auxiliary RV $\gamma_{u}$ in (41) is defined as $\gamma_{u} \triangleq \min _{\substack{1 \leq i \leq N_{s} \\ i \neq j}}\left\{\gamma_{g_{i}}\right\}$, and is thus an exponentially distributed RV with mean $\bar{\gamma}_{u}=\left(\sum_{\substack{i=1 \\ i \neq j}}^{N_{s}} \bar{\gamma}_{g_{i}}^{-1}\right)^{-1}$. Based on the definition of $\gamma_{m}$, we therefore have $\gamma_{m}=\min \left\{\gamma_{g_{j}}, \gamma_{u}, \gamma_{R}\right\}$. It can be shown that among the three possible cases $\gamma_{m}=\gamma_{R}, \gamma_{m}=\gamma_{u}$, and 
$\gamma_{m}=\gamma_{g_{j}}$, the latter dominates the asymptotic behavior of $\Psi_{i}(s)$ (the proof is omitted due to space limitations). Consequently, we can write $\Psi_{i}(s) \stackrel{\circ}{=} \Psi_{i}^{1}(s)+\Psi_{i}^{2}(s)$, where $\Psi_{i}^{1}(s)$ and $\Psi_{i}^{2}(s)$ correspond to the two cases $\gamma_{g_{j}} \leq \gamma_{R} \leq \gamma_{u}$ and $\gamma_{g_{j}} \leq \gamma_{u} \leq \gamma_{R}$, respectively, and are defined as

$$
\Psi_{i}^{1}(s, \theta) \triangleq \frac{d_{R}^{2 i}}{\bar{\gamma}_{g_{j}} \bar{\gamma}_{R} \bar{\gamma}_{u}} \int_{0}^{\infty} \mathrm{d} \gamma_{g_{j}} \mathrm{e}^{-\gamma_{g_{j}}\left(\bar{d}_{R}\left(\hat{x}_{R}\right) s+\frac{\alpha}{\sin ^{2} \theta}+\frac{1}{\bar{\gamma}_{j}}\right)} \gamma_{g_{j}}^{2 i} \int_{\gamma_{g_{j}}}^{\infty} \mathrm{d} \gamma_{u} \mathrm{e}^{-\gamma_{u} / \bar{\gamma}_{u}} \int_{\gamma_{g_{j}}}^{\gamma_{u}} \mathrm{~d} \gamma_{R} \mathrm{e}^{-\gamma_{R} / \bar{\gamma}_{R}} \gamma_{R}^{-i}
$$

and

$$
\Psi_{i}^{2}(s, \theta) \triangleq \frac{d_{R}^{2 i}}{\bar{\gamma}_{g_{j}} \bar{\gamma}_{R} \bar{\gamma}_{u}} \int_{0}^{\infty} \mathrm{d} \gamma_{g_{j}} \mathrm{e}^{-\gamma_{g_{j}}\left(\bar{d}_{R}\left(\hat{x}_{R}\right) s+\frac{\alpha}{\sin ^{2} \theta}+\frac{1}{\bar{\gamma}_{j}}\right)} \gamma_{g_{j}}^{2 i} \int_{\gamma_{g_{j}}}^{\infty} \mathrm{d} \gamma_{u} \mathrm{e}^{-\gamma_{u} / \bar{\gamma}_{u}} \int_{\gamma_{u}}^{\infty} \mathrm{d} \gamma_{R} \mathrm{e}^{-\gamma_{R} / \bar{\gamma}_{R}} \gamma_{R}^{-i}
$$

In the following, we investigate the asymptotic behavior of $\Psi_{i}^{1}(s, \theta)$ and $\Psi_{i}^{2}(s, \theta)$ for $\bar{\gamma}_{g_{j}}, \bar{\gamma}_{u}, \bar{\gamma}_{R} \rightarrow \infty$, respectively. For $\Psi_{i}^{1}(s, \theta)$, according to (42), we can write

$$
\begin{aligned}
\Psi_{i}^{1}(s, \theta)=\frac{d_{R}^{2 i}}{\bar{\gamma}_{g_{j}} \bar{\gamma}_{R} \bar{\gamma}_{u}} \int_{0}^{\infty} \mathrm{d} \gamma_{g_{j}} \mathrm{e}^{-\gamma_{g_{j}}\left(\bar{d}_{R}\left(\hat{x}_{R}\right) s+\frac{\alpha}{\sin ^{2} \theta}+1 / \bar{\gamma}_{g_{j}}\right)} \gamma_{g_{j}}^{2 i} \\
\times \int_{\gamma_{g_{j}}}^{\infty} \mathrm{d} \gamma_{u} \mathrm{e}^{-\gamma_{u} / \bar{\gamma}_{u}}\left[\bar{\gamma}_{R}^{1-i} \Gamma\left(1-i, \gamma_{g_{j}} / \bar{\gamma}_{R}\right)-\bar{\gamma}_{R}^{1-i} \Gamma\left(1-i, \gamma_{u} / \bar{\gamma}_{R}\right)\right]
\end{aligned}
$$

To determine the asymptotic behavior of $\Psi_{i}^{1}(s, \theta)$ we consider the three cases $i>1, i=1$, and $i=0$, respectively, and exploit the asymptotic properties of the incomplete Gamma function $\Gamma(\cdot, z)$ for $z \rightarrow 0$ [19]

$$
\Gamma(-\kappa, z) \doteq \begin{cases}\frac{(-1)^{\kappa}}{\kappa !}(\psi(\kappa+1)-\log z)+\frac{z^{-\kappa}}{\kappa} & \kappa \geq 1 \\ -\log z-\gamma & \kappa=0\end{cases}
$$

In particular, for $i>1$ from (45) we have $\Gamma\left(1-i, \gamma_{g_{j}} / \bar{\gamma}_{R}\right) \stackrel{\circ}{=} 1 /(i-1)\left(\gamma_{g_{j}} / \bar{\gamma}_{R}\right)^{1-i}$. Therefore, (44) reduces to

$$
\begin{aligned}
\Psi_{i}^{1}(s, \theta) & \stackrel{\circ}{=} \frac{d_{R}^{2 i}}{\bar{\gamma}_{g_{j}} \bar{\gamma}_{R} \bar{\gamma}_{u}(i-1)} \int_{0}^{\infty} \mathrm{d} \gamma_{g_{j}} \mathrm{e}^{-\gamma_{g_{j}}\left(\bar{d}_{R}\left(\hat{x}_{R}\right) s+\frac{\alpha}{\sin ^{2} \theta}+1 / \bar{\gamma}_{g_{j}}\right)} \gamma_{g_{j}}^{2 i}\left(\gamma_{g_{j}}^{1-i} \bar{\gamma}_{u}-\bar{\gamma}_{u}^{2-i} \Gamma\left(2-i, \gamma_{g_{j}} / \bar{\gamma}_{u}\right)\right) \\
& \stackrel{\circ}{=}\left(\bar{\gamma}_{g_{j}}^{-1} \bar{\gamma}_{R}^{-1}\right),
\end{aligned}
$$

where we have again used (45) to obtain the last asymptotic equality. For $i=1$, we have $\Gamma\left(0, \gamma_{g_{j}} / \bar{\gamma}_{R}\right) \stackrel{\circ}{=}-\log \left(\gamma_{g_{j}} / \bar{\gamma}_{R}\right)$, and therefore, (44) can be written as

$$
\begin{aligned}
\Psi_{i}^{1}(s, \theta) & =\frac{d_{R}^{2 i}}{\bar{\gamma}_{g_{j}} \bar{\gamma}_{R} \bar{\gamma}_{u}} \int_{0}^{\infty} \mathrm{d} \gamma_{g_{j}} \mathrm{e}^{-\gamma_{g_{j}}\left(\bar{d}_{R}\left(\hat{x}_{R}\right) s+\frac{\alpha}{\sin ^{2} \theta}+1 / \bar{\gamma}_{g_{j}}\right)} \gamma_{g_{j}}^{2}\left[\int_{\gamma_{g_{j}}}^{\infty} \mathrm{d} \gamma_{u} \log \gamma_{u} \mathrm{e}^{-\gamma_{u} / \bar{\gamma}_{u}}-\log \left(\gamma_{g_{j}}\right) \bar{\gamma}_{u}\right] \\
& \stackrel{\circ}{=}\left(\bar{\gamma}_{g_{j}}^{-1} \bar{\gamma}_{R}^{-1} \log \left(\bar{\gamma}_{u}\right)\right) .
\end{aligned}
$$

Finally, for $i=0, \Gamma\left(1, \gamma_{\mathrm{eq}} / \bar{\gamma}_{R}\right) \stackrel{\circ}{=}$ is valid and therefore after using an appropriate transformation of variables in (42), we arrive at

$$
\begin{aligned}
\Psi_{i}^{1}(s, \theta) & =\frac{1}{\bar{\gamma}_{g_{j}} \bar{\gamma}_{R} \bar{\gamma}_{u}} \int_{0}^{\infty} \mathrm{d} \gamma_{u} \int_{0}^{\gamma_{u}} \mathrm{~d} \gamma_{R} \int_{0}^{\gamma_{R}} \mathrm{~d} \gamma_{g_{j}} \mathrm{e}^{-\left(\gamma_{g_{j}} \bar{d}_{R}\left(\hat{x}_{R}\right) s+\frac{\alpha \gamma_{g_{j}}}{\sin ^{2} \theta}\right)} \mathrm{e}^{-\gamma_{g_{j}} / \bar{\gamma}_{g_{j}}} \mathrm{e}^{-\gamma_{R} / \bar{\gamma}_{R}} \mathrm{e}^{-\gamma_{u} / \bar{\gamma}_{u}} \\
& =\frac{\bar{\gamma}_{u}}{\bar{\gamma}_{g_{j}}\left(\bar{\gamma}_{R}+\bar{\gamma}_{u}\right)\left(\bar{d}_{R}\left(\hat{x}_{R}\right) s+\frac{\alpha}{\sin ^{2} \theta}\right)} .
\end{aligned}
$$


For $\Psi_{i}^{2}(s, \theta)$, we first write (43) as

$$
\Psi_{i}^{2}(s, \theta)=\frac{d_{R}^{2 i}}{\bar{\gamma}_{g_{j}} \bar{\gamma}_{R} \bar{\gamma}_{u}} \int_{0}^{\infty} \mathrm{d} \gamma_{g_{j}} \mathrm{e}^{-\gamma_{g_{j}}\left(\bar{d}_{R}\left(\hat{x}_{R}\right) s+\frac{\alpha}{\sin ^{2} \theta}+1 / \bar{\gamma}_{g_{j}}\right)} \gamma_{g_{j}}^{2 i} \int_{\gamma_{g_{j}}}^{\infty} \mathrm{d} \gamma_{u} \mathrm{e}^{-\gamma_{u} / \bar{\gamma}_{u}} \bar{\gamma}_{R}^{1-i} \Gamma\left(1-i, \gamma_{u} / \bar{\gamma}_{R}\right)
$$

Using an approach similar to that used in obtaining the asymptotic $\Psi_{i}^{1}(s, \theta)$, for $i>1$, we have

$$
\Psi_{i}^{2}(s, \theta) \stackrel{\circ}{=} \frac{d_{R}^{2 i}}{\bar{\gamma}_{g_{j}} \bar{\gamma}_{R} \bar{\gamma}_{u}(i-1)} \int_{0}^{\infty} \mathrm{d} \gamma_{g_{j}} \mathrm{e}^{-\gamma_{g_{j}}\left(\bar{d}_{R}\left(\hat{x}_{R}\right) s+\frac{\alpha}{\sin ^{2} \theta}+1 / \bar{\gamma}_{g_{j}}\right)} \gamma_{g_{j}}^{2 i}\left(\bar{\gamma}_{u}^{2-i} \Gamma\left(2-i, \gamma_{g_{j}} / \bar{\gamma}_{u}\right)\right),
$$

which leads to $\Psi_{i}^{2}(s, \theta) \stackrel{\circ}{=}\left(\bar{\gamma}_{g_{j}}^{-1} \bar{\gamma}_{R}^{-1} \bar{\gamma}_{u}^{-1}\right)$ for $i>2$ and $\Psi_{i}^{2}(s, \theta) \stackrel{\circ}{=}\left(\bar{\gamma}_{g_{j}}^{-1} \bar{\gamma}_{R}^{-1} \bar{\gamma}_{u}^{-1} \log \left(\bar{\gamma}_{u}\right)\right)$ for $i=2$. Furthermore, for $i=1$ and $i=0$, we obtain

$$
\begin{aligned}
\Psi_{i}^{2}(s, \theta) & =\frac{d_{R}^{2 i}}{\bar{\gamma}_{g_{j}} \bar{\gamma}_{R} \bar{\gamma}_{u}} \int_{0}^{\infty} \mathrm{d} \gamma_{g_{j}} \mathrm{e}^{-\gamma_{g_{j}}\left(\bar{d}_{R}\left(\hat{x}_{R}\right) s+\frac{\alpha}{\sin ^{2} \theta}+1 / \bar{\gamma}_{g_{j}}\right)} \gamma_{g_{j}}^{2}\left[\int_{\gamma_{g_{j}}}^{\infty} \mathrm{d} \gamma_{u} \mathrm{e}^{-\gamma_{u} / \bar{\gamma}_{u}} \log \left(\gamma_{u}\right)-\bar{\gamma}_{u} \log \left(\bar{\gamma}_{R}\right)\right] \\
& \stackrel{\circ}{=}\left(\bar{\gamma}_{g_{j}}^{-1} \bar{\gamma}_{R}^{-1}\right)
\end{aligned}
$$

and

$$
\begin{aligned}
\Psi_{i}^{2}(s, \theta) & =\frac{1}{\bar{\gamma}_{g_{j}} \bar{\gamma}_{R} \bar{\gamma}_{u}} \int_{0}^{\infty} \mathrm{d} \gamma_{R} \int_{0}^{\gamma_{R}} \mathrm{~d} \gamma_{u} \int_{0}^{\gamma_{u}} \mathrm{~d} \gamma_{g_{j}} \mathrm{e}^{-\gamma_{g_{j}}\left(\bar{d}_{R}\left(\hat{x}_{R}\right) s+\frac{\alpha}{\sin ^{2} \theta}+\frac{1}{\bar{\gamma}_{g_{j}}}\right)} \mathrm{e}^{-\gamma_{R} / \bar{\gamma}_{R}} \mathrm{e}^{-\gamma_{u} / \bar{\gamma}_{u}} \\
& \stackrel{\bar{\gamma}_{R}}{\bar{\gamma}_{g_{j}}\left(\bar{\gamma}_{R}+\bar{\gamma}_{u}\right)\left(\bar{d}_{R}\left(\hat{x}_{R}\right) s+\frac{\alpha}{\sin ^{2} \theta}\right)},
\end{aligned}
$$

respectively. As a result, based on (46)-(48) and (50)-(52) we obtain $\Psi_{i}(s, \theta) \stackrel{\circ}{=} \Psi_{i}^{1}(s, \theta)+\Psi_{i}^{2}(s, \theta)$ as

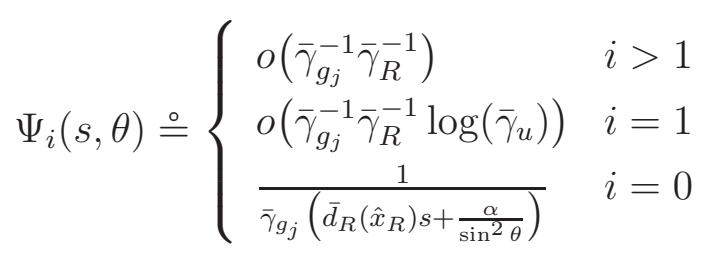

Substituting this result into (40) leads to (37) upon using (38).

Lemma 3: The asymptotic behavior of $I(s) \triangleq \mathcal{E}_{\gamma_{\text {eq }}, \gamma_{R}, \bar{n}_{D, R}}\left\{\mathrm{e}^{-s \lambda_{R} \Delta_{R}\left(x_{R}, \tilde{x}_{R}, x_{R}\right)}\right\}$ for $\bar{\gamma}_{g_{i}} \rightarrow \infty, 1 \leq$ $i \leq N_{s}, \bar{\gamma}_{R} \rightarrow \infty$ is given by

$$
I(s) \stackrel{\circ}{=} \frac{1}{\bar{\gamma}_{\mathrm{eq}} d_{R}^{2} s}-\frac{1}{\bar{\gamma}_{R} d_{R}^{2} s(s-1)}
$$

for $d_{R} \neq 0$, while $I(s)=1$ is valid for $d_{R}=0$.

Proof. Since from (39) we have $\lambda_{R} \Delta_{R}\left(x_{R}, \tilde{x}_{R}, x_{R}\right)=\gamma_{m} d_{R}^{2}+\frac{2 \gamma_{m}}{\sqrt{\gamma_{R}}} d_{R} \Re\left\{\bar{n}_{D, R}^{*}\right\}$, we conclude that $I(s)=1$ is valid for $d_{R}=0$. For $d_{R} \neq 0$ we use Taylor series expansion $\mathrm{e}^{x}=\sum_{i=0}^{\infty} x^{i} / i$ ! to write $I\left(s \mid \bar{n}_{D, R}\right) \triangleq \mathcal{E}_{\gamma_{\mathrm{eq}}, \gamma_{R}}\left\{\mathrm{e}^{-s \lambda_{R} \Delta_{R}\left(x_{R}, \tilde{x}_{R}, x_{R}\right)}\right\}$ as

$$
I\left(s \mid \bar{n}_{D, R}\right)=\mathcal{E}_{\bar{n}_{D, R}}\left\{\sum_{i=0}^{\infty} \frac{2^{i} \eta_{i}}{(2 i) !}\left|\bar{n}_{D, R}\right|^{2 i} s^{2 i} \Upsilon_{i}(s, \theta)\right\}
$$


where

$$
\Upsilon_{i}(s, \theta) \triangleq \mathcal{E}_{\gamma_{\mathrm{eq}}, \gamma_{R}}\left\{\mathrm{e}^{-\gamma_{m} d_{R}^{2} s}\left(\frac{\gamma_{m} d_{R}}{\sqrt{\gamma_{R}}}\right)^{2 i}\right\}=\frac{d_{R}^{2 i}}{\bar{\gamma}_{\mathrm{eq}} \bar{\gamma}_{R}} \int_{0}^{\infty} \int_{0}^{\infty} \mathrm{e}^{-\gamma_{m} d_{R}^{2} s} \gamma_{m}^{2 i} \gamma_{R}^{-i} \mathrm{e}^{-\gamma_{\mathrm{eq}} / \bar{\gamma}_{\mathrm{eq}}} \mathrm{e}^{-\gamma_{R} / \bar{\gamma}_{R}} \mathrm{~d} \gamma_{\mathrm{eq}} \mathrm{d} \gamma_{R} .
$$

Splitting the inner integration interval in (56) into two intervals $\left[0, \gamma_{\mathrm{eq}}\right),\left[\gamma_{\mathrm{eq}}, \infty\right)$ yields $\Upsilon_{i}(s, \theta)=$ $\Upsilon_{i}^{1}(s, \theta)+\Upsilon_{i}^{2}(s, \theta)$ where

$$
\Upsilon_{i}^{1}(s, \theta) \triangleq \frac{d_{R}^{2 i}}{\bar{\gamma}_{\mathrm{eq}} \bar{\gamma}_{R}} \int_{0}^{\infty} \mathrm{d} \gamma_{\mathrm{eq}} \mathrm{e}^{-\gamma_{\mathrm{eq}} / \bar{\gamma}_{\mathrm{eq}}} \int_{0}^{\gamma_{\mathrm{eq}}} \mathrm{d} \gamma_{R} \gamma_{R}^{i} \mathrm{e}^{-\left(\gamma_{R} d_{R}^{2} s+\gamma_{R} / \bar{\gamma}_{R}\right)}
$$

and

$$
\Upsilon_{i}^{2}(s, \theta) \triangleq \frac{d_{R}^{2 i}}{\bar{\gamma}_{\mathrm{eq}} \bar{\gamma}_{R}} \int_{0}^{\infty} \mathrm{d} \gamma_{\mathrm{eq}} \gamma_{\mathrm{eq}}^{2 i} \mathrm{e}^{-\gamma_{\mathrm{eq}}\left(d_{R}^{2} s+1 / \bar{\gamma}_{\mathrm{eq}}\right)} \int_{\gamma_{\mathrm{eq}}}^{\infty} \mathrm{d} \gamma_{R} \gamma_{R}^{-i} \mathrm{e}^{-\left(\gamma_{R} / \bar{\gamma}_{R}\right)}
$$

In the following, we determine the asymptotic behavior of $\Upsilon_{i}^{1}(s, \theta)$ and $\Upsilon_{i}^{2}(s, \theta)$, respectively, for $\bar{\gamma}_{\text {eq }}, \bar{\gamma}_{R} \rightarrow \infty$. For $\Upsilon_{i}^{1}(s, \theta)$, we write (57) as

$$
\Upsilon_{i}^{1}(s, \theta)=\frac{i !}{\bar{\gamma}_{R} d_{R}^{2} s^{i+1}}-\frac{d_{R}^{2 i}}{\bar{\gamma}_{\mathrm{eq}} \bar{\gamma}_{R}} \sum_{k=0}^{i} \int_{0}^{\infty} \frac{i ! \gamma_{\mathrm{eq}}^{k} \mathrm{e}^{\left(d_{R}^{2} s+1 / \bar{\gamma}_{R}+1 / \bar{\gamma}_{\mathrm{eq}}\right) \gamma_{\mathrm{eq}}}}{k !\left(d_{R}^{2} s+1 / \bar{\gamma}_{R}\right)^{i-k+1}} \mathrm{~d} \gamma_{\mathrm{eq}} \stackrel{\circ}{=} \frac{i !}{\bar{\gamma}_{R} d_{R}^{2} s^{i+1}} .
$$

For $\Upsilon_{i}^{2}(s, \theta)$, we first express (58) as

$$
\Upsilon_{i}^{2}(s, \theta)=\frac{d_{R}^{i}}{\bar{\gamma}_{\mathrm{eq}} \bar{\gamma}_{R}^{i}} \int_{0}^{\infty} \mathrm{d} \gamma_{\mathrm{eq}} \gamma_{\mathrm{eq}}^{2 i} \mathrm{e}^{-\gamma_{\mathrm{eq}}\left(d_{R}^{2} s+1 / \bar{\gamma}_{\mathrm{eq}}\right)} \Gamma\left(1-i, \gamma_{\mathrm{eq}} / \bar{\gamma}_{R}\right)
$$

Following steps similar to those used in Lemma 2 to obtain the asymptotic behavior of $\Psi_{i}^{2}(s, \theta)$ we arrive at

$$
\Upsilon_{i}^{2}(s, \theta) \doteq \begin{cases}o\left(\bar{\gamma}_{\mathrm{eq}}^{-1} \bar{\gamma}_{R}^{-1}\right) & i>1 \\ \frac{2 \log \left(\bar{\gamma}_{R}\right)}{d_{R}^{6} s^{3} \bar{\gamma}_{\mathrm{eq}} \bar{\gamma}_{R}} & i=1 \\ \frac{1}{\bar{\gamma}_{\mathrm{eq}} d_{R}^{2} s} & i=0\end{cases}
$$

For $\Upsilon_{i}(s, \theta)=\Upsilon_{i}^{1}(s, \theta)+\Upsilon_{i}^{2}(s, \theta)$, we get with (59) and (61)

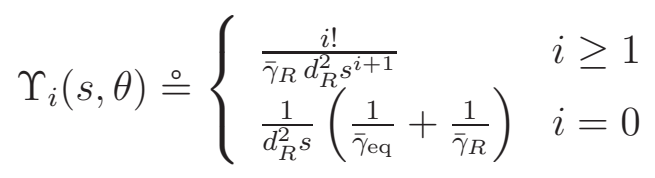

Substituting (62) in (55) results in

$$
I\left(s \mid \bar{n}_{D, R}\right)=\frac{1}{d_{R}^{2} s}\left(\frac{1}{\bar{\gamma}_{\mathrm{eq}}}+\frac{1}{\bar{\gamma}_{R}}\right)+\frac{1}{d_{R}^{2} s \bar{\gamma}_{R}} \sum_{i=1}^{\infty} \frac{2^{i} i ! \eta_{i}}{(2 i) !}\left|\bar{n}_{D, R}\right|^{2 i} s^{i}=\frac{1}{d_{R}^{2} s \bar{\gamma}_{\mathrm{eq}}}+\frac{\mathrm{e}^{\left|\bar{n}_{D, R}\right|^{2} s}}{d_{R}^{2} s \bar{\gamma}_{R}}
$$

where we have used $\eta_{i}=\frac{(2 i) !}{2^{i}(i !)^{2}}$. Finally, averaging $I\left(s \mid \bar{n}_{D, R}\right)$ over the Rayleigh distributed RV $\left|\bar{n}_{D, R}\right|$ leads to (54). 
Lemma 4: The asymptotic behavior of $\Phi_{R}^{c}(s) \triangleq \mathcal{E}_{\gamma_{\mathrm{eq}}, \gamma_{R}, \bar{n}_{D, R}}\left\{\left(1-\beta Q\left(\sqrt{2 \alpha \gamma_{\mathrm{eq}}}\right)\right) e^{-s \lambda_{R} \Delta_{R}\left(x_{R}, \tilde{x}_{R}, x_{R}\right)}\right\}$ for $\bar{\gamma}_{g_{i}} \rightarrow \infty, 1 \leq i \leq N_{s}, \bar{\gamma}_{R} \rightarrow \infty$ is given by

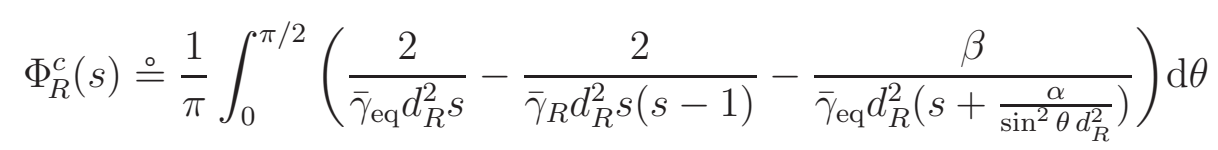

for $d_{R} \neq 0$, while $\Phi_{R}^{c}(s) \stackrel{\circ}{=}$ is valid for $d_{R}=0$.

Proof. We first note that $\Phi_{R}^{c}(s)=I(s)-\sum_{j=1}^{N_{s}} \Phi_{R, j}^{e}\left(x_{R} ; s\right)$, where we have employed $Q\left(\sqrt{2 \alpha \gamma_{\mathrm{eq}}}\right) \approx$ $\sum_{i=1}^{N_{s}} Q\left(\sqrt{2 \alpha \gamma_{g_{i}}}\right)$ which is valid for $\bar{\gamma}_{g_{i}} \rightarrow \infty, 1 \leq i \leq N_{s}$. For $d_{R} \neq 0$, combining (37) and (54)

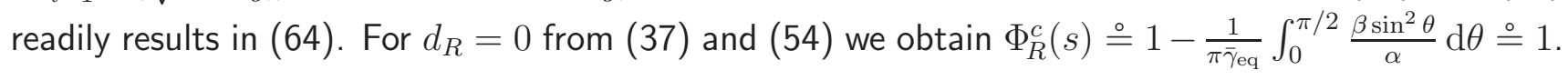

Proof. [Proposition 1] Based on Lemma 1, $\Phi_{f_{i}}(s)$ can be written as $\Phi_{f_{i}}(s) \stackrel{\circ}{=} \tilde{k}_{1} / \bar{\gamma}$ for $x_{i} \neq \tilde{x}_{i}$ and $\Phi_{f_{i}}(s) \stackrel{\circ}{=}$ for $x_{i}=\tilde{x}_{i}$, where $\tilde{k}_{1}$ is a finite (positive) constant. Furthermore, using Lemmas 2 and

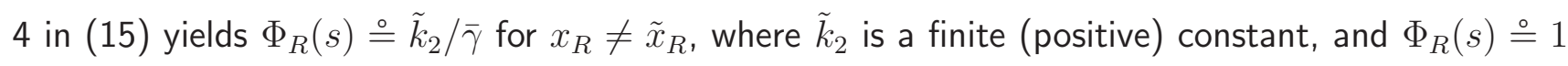
for $x_{R}=\tilde{x}_{R}$. Therefore, based on (13) we conclude that $G_{d, \mathrm{PEP}}$ is given by the number of non-zero elements of vector $\left[x_{1}-\tilde{x}_{1}, \cdots, x_{N_{s}}-\tilde{x}_{N_{s}}, x_{R}-\tilde{x}_{R}\right]^{T}$. Since $\mu_{\mathcal{X}}: \mathcal{A} \rightarrow \mathcal{X}$ is a one-to-one mapping function, $G_{d, \mathrm{PEP}}$ is alternatively given by the Hamming distance between the transmit symbol vectors $\boldsymbol{s}_{e}$ and $\tilde{\boldsymbol{s}}_{e}$ denoted by $d_{H}(\boldsymbol{x}, \tilde{\boldsymbol{x}})$. To show that $d_{H}(\boldsymbol{x}, \tilde{\boldsymbol{x}}) \geq 2$, we first note that by definition we have $\boldsymbol{x} \neq \tilde{\boldsymbol{x}}$, and therefore $s_{i} \neq \tilde{s}_{i}$ is valid for $i \in \mathcal{I}$, where $\mathcal{I}$ is a non-empty index set. For $|\mathcal{I}| \geq 2, d_{H}(\boldsymbol{x}, \tilde{\boldsymbol{x}}) \geq 2$ immediately follows. For $|\mathcal{I}|=1$ it is easy to see that $s_{R} \neq \tilde{s}_{R}$, resulting in $d_{H}(\boldsymbol{x}, \tilde{\boldsymbol{x}})=2$.

Proof. [Proposition 2] For a given transmit signal vector $\boldsymbol{x}$, set $\mathcal{C}_{i}(\boldsymbol{x})$ in (26) can be partitioned into $N_{s}$ disjoints sets $\mathcal{C}_{i}^{l}(\boldsymbol{x}), 1 \leq l \leq N_{s}$, i.e., $\mathcal{C}_{i}(\boldsymbol{x})=\bigcup_{l=1}^{N_{s}} \mathcal{C}_{i}^{l}(\boldsymbol{x})$, where $\mathcal{C}_{i}^{l}(\boldsymbol{x})$ is defined in (30). Therefore, using (5) and (26) the asymptotic SER can be approximated as

$$
P_{s}^{i} \stackrel{\circ}{=} \frac{1}{M^{N_{s}}} \sum_{\boldsymbol{x} \in \mathcal{X}^{N_{s}}} \sum_{l=1}^{N_{s}} \sum_{\tilde{\boldsymbol{x}} \in \mathcal{C}_{i}^{l}(\boldsymbol{x})} P(\boldsymbol{x} \rightarrow \tilde{\boldsymbol{x}}) .
$$

For $\tilde{\boldsymbol{x}} \in \mathcal{C}_{i}^{i}(\boldsymbol{x})$, the asymptotic PEP can be obtained from (20) and (22) as

$$
P(\boldsymbol{x} \rightarrow \tilde{\boldsymbol{x}}) \stackrel{\circ}{=} \frac{1}{\bar{\gamma}_{f_{i}}}\left(\phi_{c}^{g}(\boldsymbol{x}, \tilde{\boldsymbol{x}}) \sum_{j=1}^{N_{s}} \frac{1}{\bar{\gamma}_{g_{j}}}+\frac{\phi_{c}^{R}(\boldsymbol{x}, \tilde{\boldsymbol{x}})}{\bar{\gamma}_{R}}\right)+\sum_{j=1}^{N_{s}} \frac{1}{\left|\mathcal{D}_{j}(\boldsymbol{x})\right|} \sum_{\hat{x}_{R} \in \mathcal{D}_{j}(\boldsymbol{x})} \frac{\phi_{e}\left(\boldsymbol{x}, \tilde{\boldsymbol{x}}, \hat{x}_{R}\right)}{\bar{\gamma}_{f_{i}} \bar{\gamma}_{g_{j}}} .
$$

For $\tilde{\boldsymbol{x}} \in \mathcal{C}_{i}^{l}(\boldsymbol{x}), l \neq i$, using (24) and (25) yields

$$
P(\boldsymbol{x} \rightarrow \tilde{\boldsymbol{x}}) \doteq \frac{\bar{\phi}_{c}(\boldsymbol{x}, \tilde{\boldsymbol{x}})}{\bar{\gamma}_{f_{i}} \bar{\gamma}_{f_{l}}}+\sum_{j=1}^{N_{s}} \frac{1}{\left|\mathcal{D}_{j}(\boldsymbol{x})\right|} \sum_{\hat{x}_{R} \in \mathcal{D}_{j}(\boldsymbol{x})} \frac{\bar{\phi}_{e}\left(\boldsymbol{x}, \tilde{\boldsymbol{x}}, \hat{x}_{R}\right)}{\bar{\gamma}_{f_{i}} \bar{\gamma}_{f_{l}} \bar{\gamma}_{g_{j}}} \doteq \frac{\bar{\phi}_{c}(\boldsymbol{x}, \tilde{\boldsymbol{x}})}{\bar{\gamma}_{f_{i}} \bar{\gamma}_{f_{l}}} .
$$

Eq. (27) can be obtained by combining (65)-(67). 


\section{References}

[1] J.N. Laneman, D.N.C. Tse, and G.W. Wornell. "Cooperative Diversity in Wireless Networks: Efficient Protocols and Outage Behavior". IEEE Trans. Inform. Theory, 50:3062-3080, December 2004.

[2] J. Boyer, D.D. Falconer, and H. Yanikomeroglu. Multihop Diversity in Wireless Relaying Channels. IEEE Trans. Commun., COM-52:1820-1830, October 2004.

[3] Y. Chen, S. Kishore, and J. Li. "Wireless Diversity Through Network Coding". In Proc. IEEE Wireless Commun. and Networking Conf. (WCNC), volume 3, pages 1681-1686, 2006.

[4] M. Yu, J. Li, and R.S. Blum. "User Cooperation Through Network Coding". In Proc. IEEE Inter. Conf. Commun. (ICC), pages 4064-4069, 2007.

[5] C. Peng, Q. Zhang, M. Zhao, Y. Yao, and W. Jia. "On the Performance Analysis of Network-Coded Cooperation in Wireless Networks". IEEE Trans. Wireless Commun., 7:3090-3097, August 2008.

[6] R. Ahlswede, N. Cai, S. Li, and R. Yeung. "Network Information Flow". IEEE Trans. Inform. Theory, 46:1204-1216, July 2000.

[7] L. Xiao, T.E. Fuja, J. Kliewer, and D.J. Costello. "A Network Coding Approach to Cooperative Diversity". IEEE Trans. Inform. Theory, 53:3714-3722, October 2007.

[8] A. Cano, T. Wang, A. Ribeiro, and G.B. Giannakis. "Link-Adaptive Distributed Coding for Multi-Source Cooperation". EURASIP J. on Adv. in Signal Process., 2008:1-12, January 2008.

[9] S. Zhang, S.C. Liew, and P.P. Lam. "Hot topic: Physical-Layer Network Coding". In Proceedings of 12th Annual International Conference on Mobile Computing and Networking (MobiCom), pages 358-365, September 2006.

[10] T. Wang and G.B. Giannakis. "Complex Field Network Coding for Multiuser Cooperative Communications". IEEE J. Select. Areas Commun., 26:561-571, April 2008.

[11] T. Wang, A. Cano, G.B. Giannakis, and J.N. Laneman. "High-Performance Cooperative Demodulation With Decode-and-Forward Relays". IEEE Trans. Commun., 55:1427-1438, July 2007.

[12] S. Lin and J.J. Costello. Error Control Coding. Prentice Hall, Englewood Cliffs, New Jersey, 1983.

[13] S. Lin, T. Kasami, T. Fujiwara, and M. Fossorier. Trellises and Trellis-Based Decoding Algorithms for Linear Block Codes. Kluwer, Norwell, MA, 1998.

[14] A.C. Ribeiro and G.B. Giannakis. Symbol Error Probabilities for General Cooperative Links. IEEE Wireless Commun., 4:1264-1273, May 2005.

[15] A. Nasri, R. Schober, and I.F. Blake. "Performance and Optimization of Cooperative Diversity Systems in Generic Noise and Interference". Submitted to the IEEE Trans. Wireless Commun., available at: http://www.ece.ubc.ca/ amirn/TW09.pdf, 2010.

[16] S. Boyd and L. Vandenberghe. Convex Optimization. U.K.: Cambridge Univ. Press, 2004.

[17] K.T. Phan, T. Le-Ngoc, S.A. Vorobyov, and C. Tellambura. "Power Allocation in Wireless Relay Networks: A Geometric Programming-Based Approach". In Proceedings of the IEEE Global Telecommun. Conf. (Globecom), pages 1-5, 2008.

[18] A. Nasri and R. Schober. "Performance of BICM-SC and BICM-OFDM Systems with Diversity Reception in Non-Gaussian Noise and Interference". IEEE Trans. Commun., pages 3316-3327, November 2009.

[19] M. Abramowitz and I. Stegun. Handbook of Mathematical Functions. Dover Publications, Inc., New York, 1970. 
Figures:

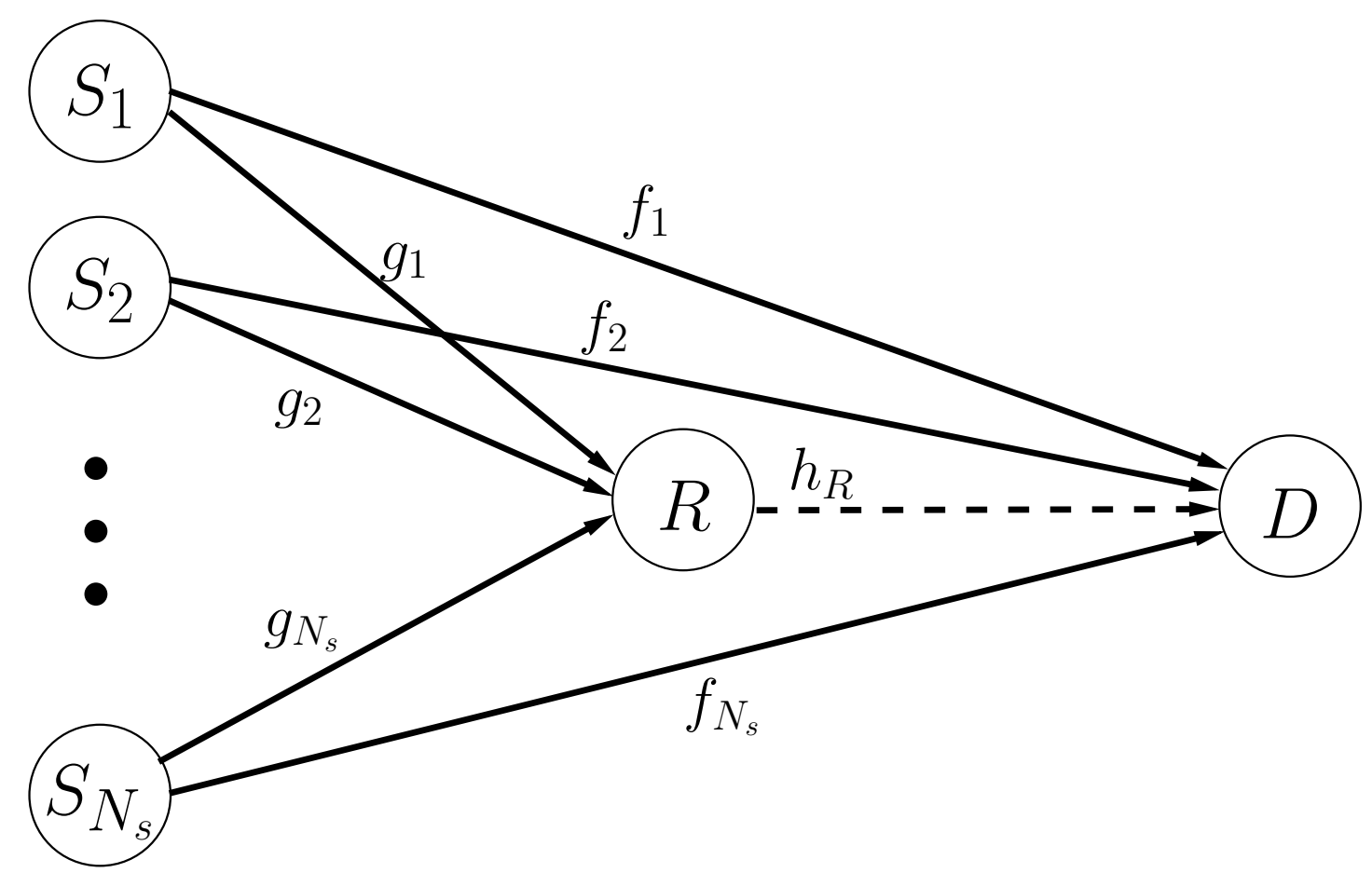

Figure 1: Block diagram of the considered NCCD system. Solid and dashed lines denote links belonging to first and second hop, respectively. 


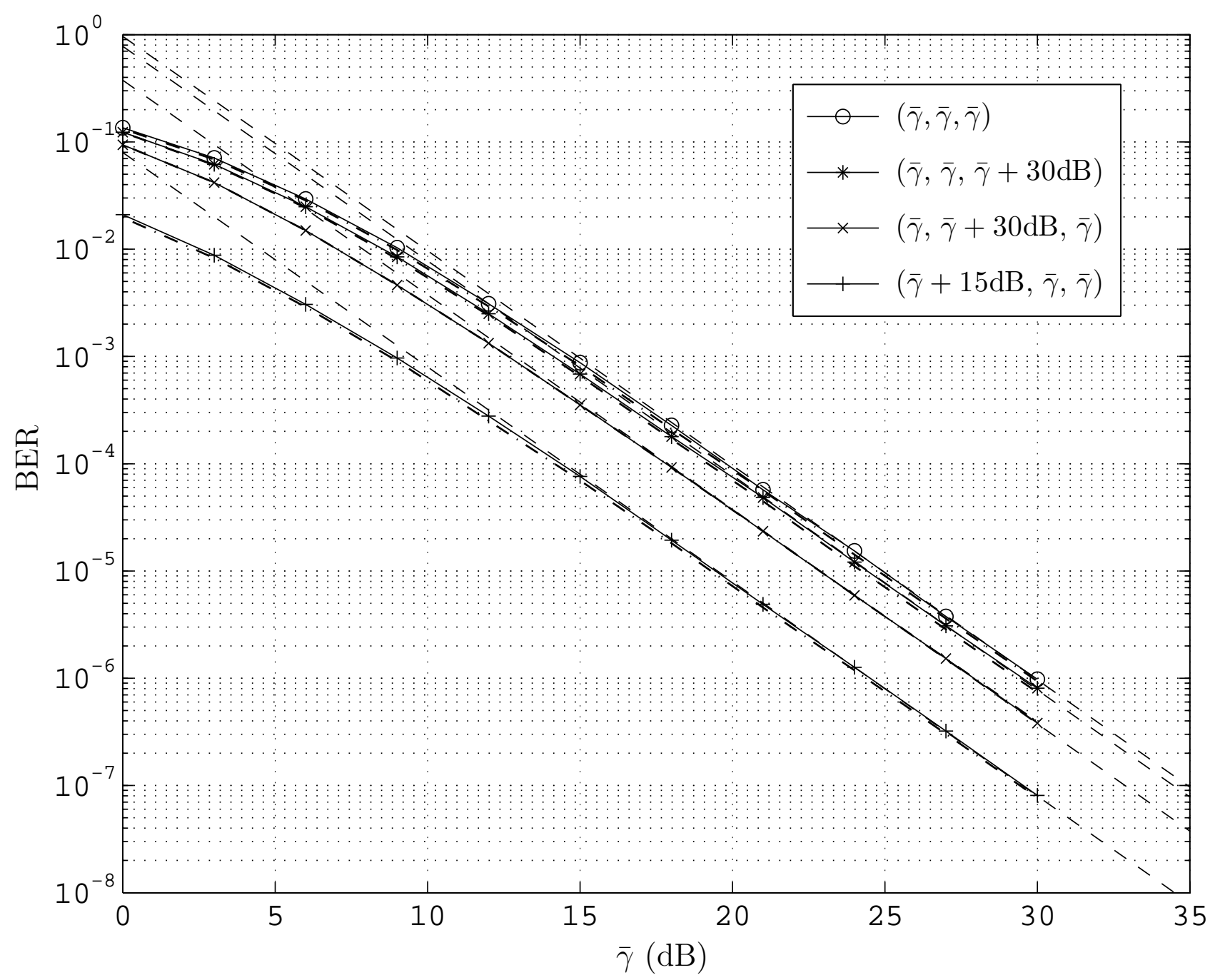

Figure 2: BER of a symmetric NCCD system with $N_{s}=2$ sources and BPSK modulation vs. $\bar{\gamma}$ for various channel quality settings $\left(\bar{\gamma}_{f}, \bar{\gamma}_{g}, \bar{\gamma}_{R}\right)$. Solid lines with markers: Simulated BER. Dashed lines: Asymptotic BER approximation. Dash-dotted lines: Simulated BER for ML combining at the destination. 


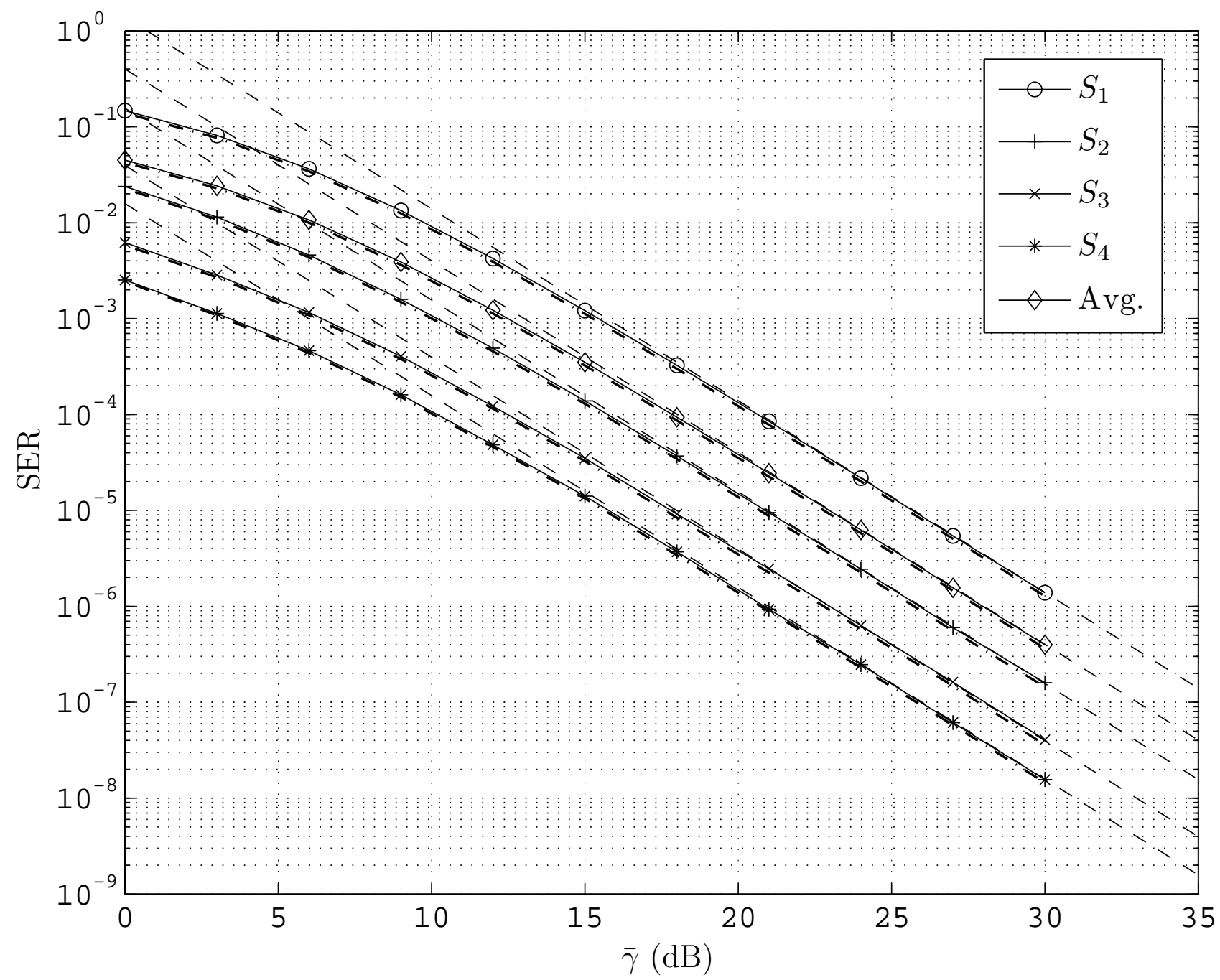

Figure 3: BER of an asymmetric NCCD system with $N_{s}=4$ sources and BPSK modulation vs. $\bar{\gamma}$. Solid lines with markers: Simulated BER. Dashed lines: Asymptotic BER approximation. Dash-dotted lines: Simulated BER for ML combining at the destination. 


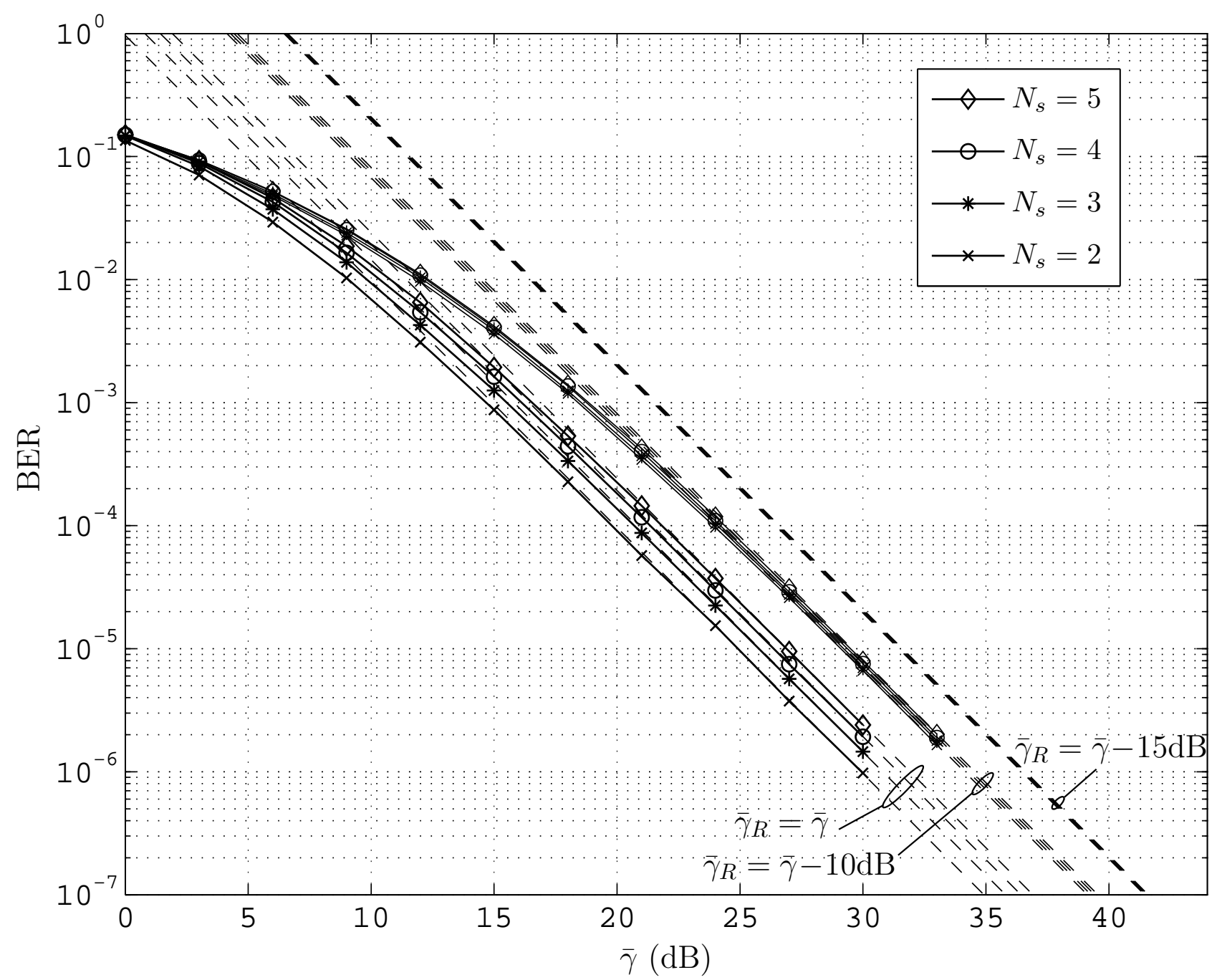

Figure 4: BER of an NCCD system with BPSK modulation vs. $\bar{\gamma}$ for different $N_{s}$. Solid lines with markers: Simulated BER. Dashed lines: Asymptotic BER approximation. 


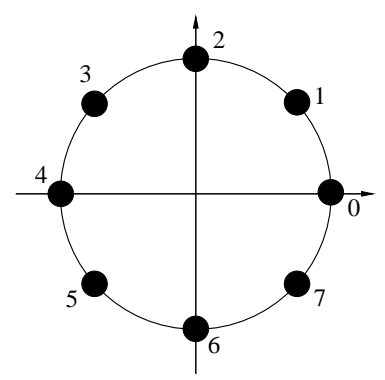

(a)

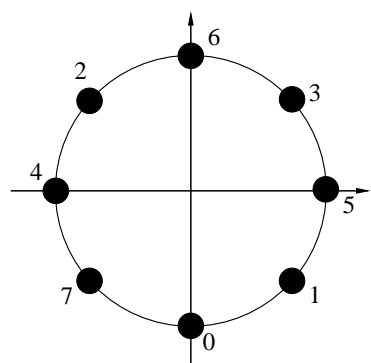

(b)

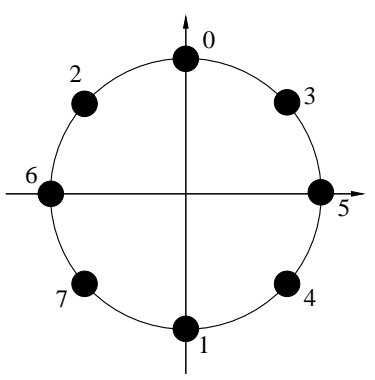

(c)

Figure 5: 8-PSK signal constellation with three different constellation mappings $\mu_{\mathcal{X}}$ : $\mathcal{A} \rightarrow \mathcal{X}$. (a) Natural mapping, (b) Optimal mapping for Case I, and (c) Optimal mapping for Case II.

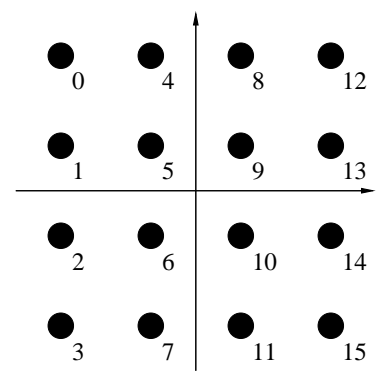

(a)

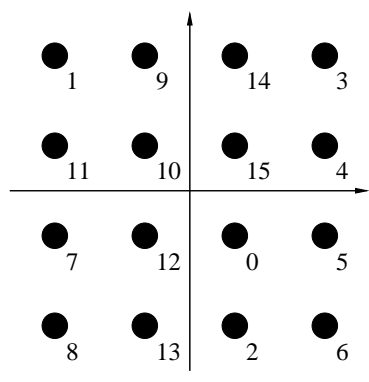

(b)

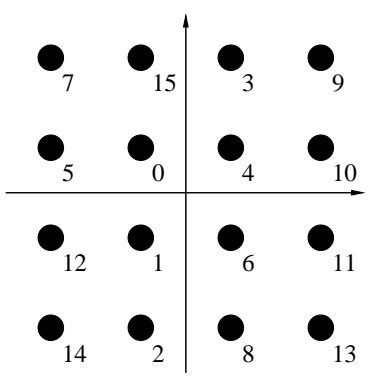

(c)

Figure 6: 16-QAM signal constellation with three different constellation mappings $\mu_{\mathcal{X}}: \mathcal{A} \rightarrow \mathcal{X}$. (a) Natural mapping, (b) Optimal mapping for Case I, and (c) Optimal mapping for Case II. 


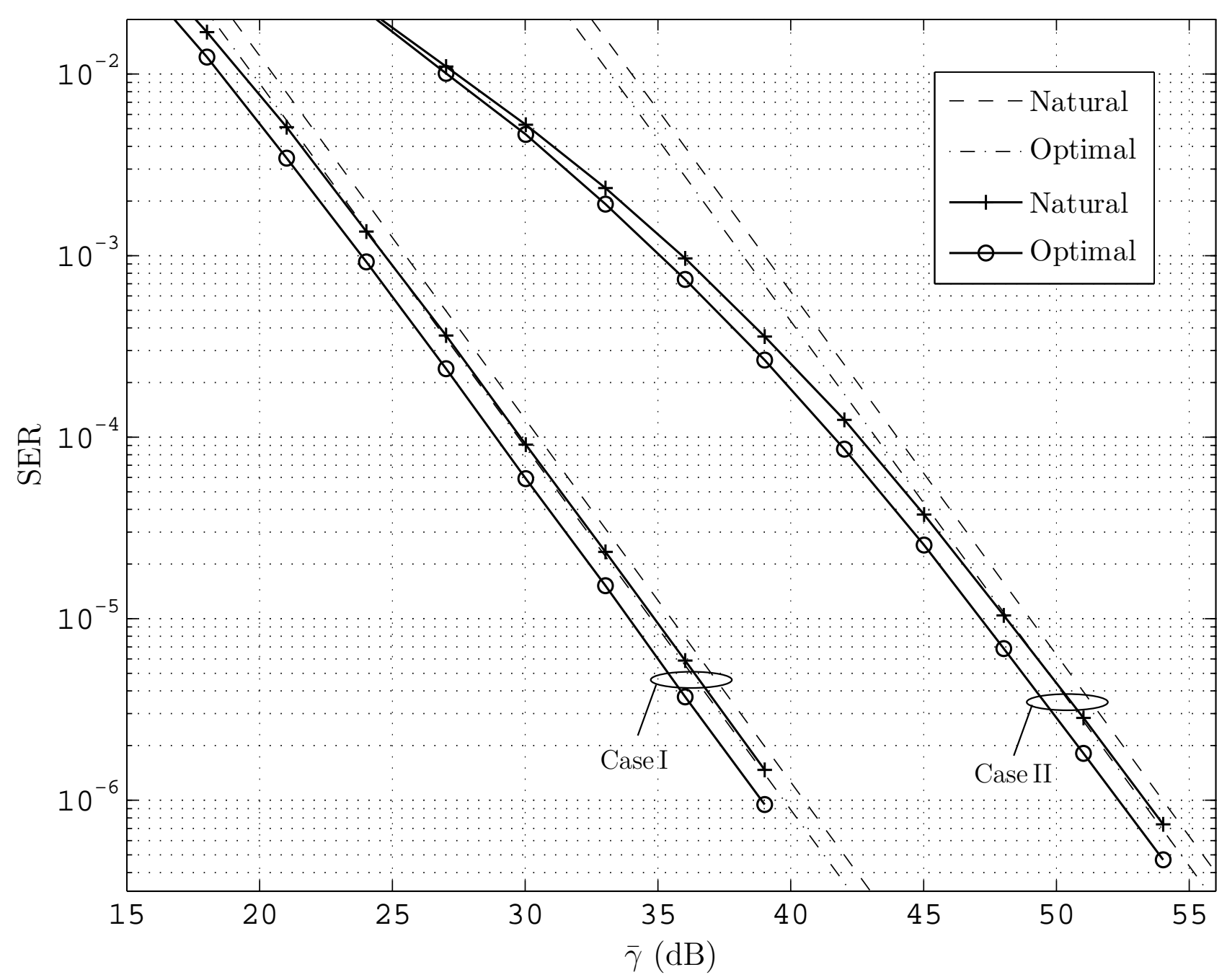

Figure 7: SER of an NCCD system with 16-QAM modulation, $N_{s}=2$, and the optimal and natural mappings depicted in Fig. 6 vs. $\bar{\gamma}$. Two channel quality settings are considered (Case I and Case II). Solid lines with markers: Simulated SER. Dashed lines: Asymptotic SER approximation for natural mapping. Dash-dotted lines: Asymptotic SER approximation for optimal mapping. 


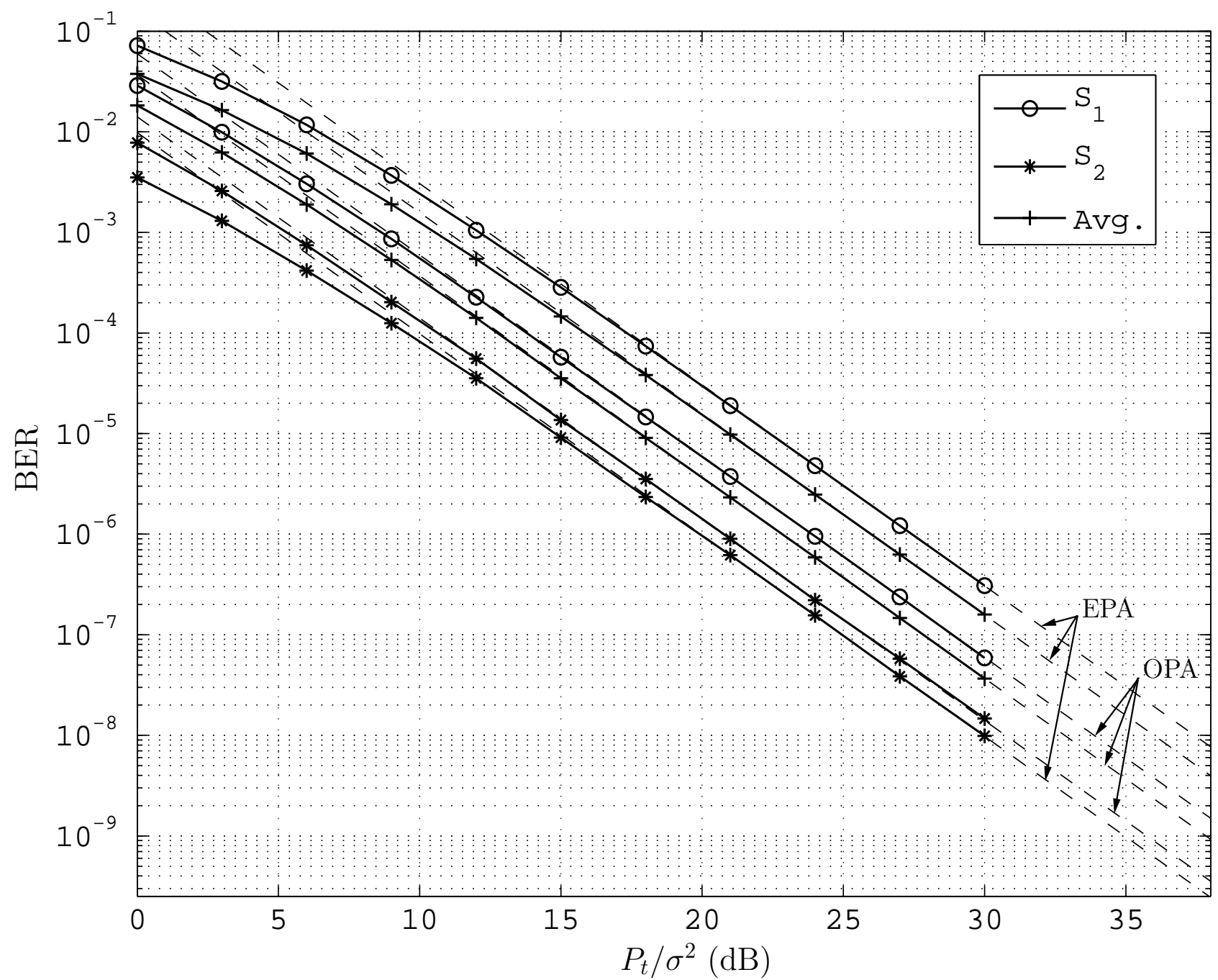

Figure 8: BER of an NCCD system with $N_{s}=2$ and BPSK modulation vs. $P_{t} / \sigma^{2}$ for max-min fair OPA and EPA. Solid lines with markers: Simulated BER. Dashed lines: Asymptotic BER approximations. 